\title{
GLOBAL WELL-POSEDNESS OF THE KP-I INITIAL-VALUE PROBLEM IN THE ENERGY SPACE
}

\author{
A. D. IONESCU, C. E. KENIG, AND D. TATARU
}

Abstract. We prove that the KP-I initial-value problem

$$
\left\{\begin{array}{l}
\partial_{t} u+\partial_{x}^{3} u-\partial_{x}^{-1} \partial_{y}^{2} u+\partial_{x}\left(u^{2} / 2\right)=0 \text { on } \mathbb{R}_{x, y}^{2} \times \mathbb{R}_{t} ; \\
u(0)=\phi
\end{array}\right.
$$

is globally well-posed in the energy space

$$
\mathbf{E}^{1}\left(\mathbb{R}^{2}\right)=\left\{\phi: \mathbb{R}^{2} \rightarrow \mathbb{R}:\|\phi\|_{\mathbf{E}^{1}\left(\mathbb{R}^{2}\right)} \approx\|\phi\|_{L^{2}}+\left\|\partial_{x} \phi\right\|_{L^{2}}+\left\|\partial_{x}^{-1} \partial_{y} \phi\right\|_{L^{2}}<\infty\right\} .
$$

\section{Contents}

1. Introduction

2. Notation and definitions

3. Global linear, bilinear and energy estimates

4. Proof of the main theorem

5. $L^{2}$ bilinear estimates

6. Energy estimates

7. Dyadic bilinear estimates, I

8. Dyadic bilinear estimates, II

References

\section{IntRoduction}

In this paper we consider the KP-I initial-value problem

$$
\left\{\begin{array}{l}
\partial_{t} u+\partial_{x}^{3} u-\partial_{x}^{-1} \partial_{y}^{2} u+\partial_{x}\left(u^{2} / 2\right)=0 ; \\
u(0)=\phi
\end{array}\right.
$$

on $\mathbb{R}_{x, y}^{2} \times \mathbb{R}_{t}$. The KP-I equation and the KP-II equation, in which the sign of the term $\partial_{x}^{-1} \partial_{y}^{2} u$ in (1.1) is + instead of - , arise in physical contexts as models for the propagation of dispersive long waves with weak transverse effects.

The first author was supported in part by an NSF grant and a Packard Fellowship. The second author was supported in part by an NSF grant. The third author was supported in part by an NSF grant. 
The KP-II equation is well understood from the point of view of well-posedness: the KP-II initial-value problem is globally well-posed for suitable data in $L^{2}$, on both $\mathbb{R}^{2}$ and $\mathbb{T}^{2}=\mathbb{S}^{1} \times \mathbb{S}^{1}$, see [4], as well as in some spaces larger than $L^{2}$, see [18] and the references therein.

On the other hand, it has been shown in [14] that the KP-I initial-value problem is badly behaved with respect to Picard iterative methods in standard Sobolev spaces, since the flow map fails to be real-analytic at the origin in these spaces 1 On the positive side, it is known that the KP-I initial value problem is globally well-posed in the "second" energy spaces on both $\mathbb{R}^{2}$ (see [11], and also [15] and [16]) and $\mathbb{T}^{2}$ (see [7]), as well as locally well-posed in larger spaces. These global well-posedness results rely on refined energy methods. In this paper we show that the KP-I initial-value problem is globally well-posed in the natural energy space of the equation.

Let $\xi, \mu$ and $\tau$ denote the Fourier variables with respect to $x, y$ and $t$ respectively. For $\sigma=1,2, \ldots$ we define the Banach spaces $\mathbf{E}^{\sigma}=\mathbf{E}^{\sigma}\left(\mathbb{R}^{2}\right)$,

$$
\mathbf{E}^{\sigma}=\left\{\phi: \mathbb{R}^{2} \rightarrow \mathbb{R}:\|\phi\|_{\mathbf{E}^{\sigma}}=\left\|\widehat{\phi}(\xi, \mu) \cdot p(\xi, \mu)(1+|\xi|)^{\sigma}\right\|_{L_{\xi, \mu}^{2}}<\infty\right\},
$$

where $\widehat{\phi}$ denotes the Fourier transform of $\phi$ and

$$
p(\xi, \mu)=1+\frac{|\mu|}{|\xi|+|\xi|^{2}} \text {. }
$$

Clearly,

$$
p(\xi, \mu)(1+|\xi|)^{\sigma}=(1+|\xi|)^{\sigma}+|\mu / \xi| \cdot(1+|\xi|)^{\sigma-1}
$$

Let

$$
\mathbf{E}^{\infty}=\bigcap_{\sigma=1}^{\infty} \mathbf{E}^{\sigma}
$$

with the induced metric. We recall the KP-I conservation laws (see, for example, [15] for formal justifications): if $t_{1}<t_{2} \in \mathbb{R} u \in C\left(\left[t_{1}, t_{2}\right]: \mathbf{E}^{\infty}\right)$ is a solution of the equation $\partial_{t} u+\partial_{x}^{3} u-\partial_{x}^{-1} \partial_{y}^{2} u+\partial_{x}\left(u^{2} / 2\right)=0$ on $\mathbb{R}^{2} \times\left(t_{1}, t_{2}\right)$ then

$$
\widetilde{E}^{0}\left(u\left(t_{1}\right)\right)=\widetilde{E}^{0}\left(u\left(t_{2}\right)\right) \text { and } \widetilde{E}^{1}\left(u\left(t_{1}\right)\right)=\widetilde{E}^{1}\left(u\left(t_{2}\right)\right),
$$

where, for any $\phi \in \mathbf{E}^{1}$,

$$
\widetilde{E}^{0}(\phi)=\int_{\mathbb{R}^{2}} \phi^{2} d x d y
$$

and

$$
\widetilde{E}^{1}(\phi)=\int_{\mathbb{R}^{2}}\left(\partial_{x} \phi\right)^{2} d x d y+\int_{\mathbb{R}^{2}}\left(\partial_{x}^{-1} \partial_{y} \phi\right)^{2} d x d y-\frac{1}{3} \int_{\mathbb{R}^{2}} \phi^{3} d x d y
$$

\footnotetext{
${ }^{1}$ Picard iterative methods can be applied, however, to produce local in time solutions for small low-regularity data in suitably weighted spaces, see [6].
} 
Consequently, if $t_{0} \in\left[t_{1}, t_{2}\right]$ and $\left\|u\left(t_{0}\right)\right\|_{\mathbf{E}^{1}} \leq 1$ then we have the uniform bound

$$
\sup _{t \in\left[t_{1}, t_{2}\right]}\|u(t)\|_{\mathbf{E}^{1}} \lesssim\left\|u\left(t_{0}\right)\right\|_{\mathbf{E}^{1}}
$$

Our main theorem concerns global well-posedness of the KP-I initial-value problem in the energy space $\mathbf{E}^{1}$.

Theorem 1.1. (a) Assume $\phi \in \mathbf{E}^{\infty}$. Then there is a unique global solution

$$
u=S^{\infty}(\phi) \in C\left(\mathbb{R}: \mathbf{E}^{\infty}\right)
$$

of the initial-value problem (1.1). In addition, for any $T \in[0, \infty)$ and any $\sigma \in$ $\{1,2, \ldots\}$

$$
\sup _{|t| \leq T}\left\|S^{\infty}(\phi)(t)\right\|_{\mathbf{E}^{\sigma}} \leq C\left(T, \sigma,\|\phi\|_{\mathbf{E}^{\sigma}}\right) .
$$

(b) Assume $T \in \mathbb{R}_{+}$. Then the mapping

$$
S_{T}^{\infty}=\mathbf{1}_{[-T, T]}(t) \cdot S^{\infty}: \mathbf{E}^{\infty} \rightarrow C\left([-T, T]: \mathbf{E}^{\infty}\right)
$$

extends uniquely to a continuous mapping

$$
S_{T}^{1}: \mathbf{E}^{1} \rightarrow C\left([-T, T]: \mathbf{E}^{1}\right)
$$

and

$$
\widetilde{E}^{j}(u(t))=\widetilde{E}^{j}(\phi) \text { for any } t \in[-T, T] \text { and } j \in\{0,1\} .
$$

We remark that the global existence of smooth solutions stated in Theorem 1.1 (a) is also new. The earlier global existence theorems of [11], [15], and [16] rely of the conservation of the second energy, which requires the stronger momentum condition $\partial_{x}^{-2} \partial_{y}^{2} \phi \in L^{2}$. Also, a simple additional argument shows that (1.8) can be improved to

$$
\sup _{|t| \leq T}\left\|S^{\infty}(\phi)(t)\right\|_{\mathbf{E}^{\sigma}} \leq C\left(T, \sigma,\|\phi\|_{\mathbf{E}^{1}}\right) \cdot\|\phi\|_{\mathbf{E}^{\sigma}}
$$

We discuss now some of the ingredients in the proof of Theorem 1.1. One might try a direct perturbative approach (which goes back to work on the KdV equation in [9], [3], [10], and nonlinear wave equations in [12]), based on the properties of solutions to the linear equation

$$
\left\{\begin{array}{l}
\partial_{t} u+\partial_{x}^{3} u-\partial_{x}^{-1} \partial_{y}^{2} u=f \\
u(0)=\phi
\end{array}\right.
$$

For some suitable spaces $\mathbf{F}^{1}(T)$ and $\mathbf{N}^{1}(T)$ one would like to prove a linear bound for solutions to (1.9) on $\mathbb{R}^{2} \times[-T, T], T \in(0,1]$, of the form

$$
\|u\|_{\mathbf{F}^{1}(T)} \lesssim\|\phi\|_{\mathbf{E}^{1}}+\|f\|_{\mathbf{N}^{1}(T)}
$$

together with a matching nonlinear estimate

$$
\left\|-\partial_{x}\left(u^{2} / 2\right)\right\|_{\mathbf{N}^{1}(T)} \lesssim\|u\|_{\mathbf{F}^{1}(T)}^{2} .
$$


Due to [14, it is known however that the inequalities (1.10), (1.11) cannot hold for any choice of the spaces $\mathbf{F}^{1}(T)$ and $\mathbf{N}^{1}(T)$; this forces us to approach the problem in a less perturbative way.

To prove Theorem 1.1 (a) we define instead the normed spaces $\mathbf{F}^{1}(T), \mathbf{N}^{1}(T)$, and the semi-normed space $\mathbf{B}^{1}(T)$ and show that if $u$ is a smooth solution of (1.1) on $\mathbb{R}^{2} \times[-T, T], T \in(0,1]$, then

$$
\left\{\begin{array}{l}
\|u\|_{\mathbf{F}^{1}(T)} \lesssim\|u\|_{\mathbf{B}^{1}(T)}+\left\|-\partial_{x}\left(u^{2} / 2\right)\right\|_{\mathbf{N}^{1}(T)} \\
\left\|-\partial_{x}\left(u^{2} / 2\right)\right\|_{\mathbf{N}^{1}(T)} \lesssim\|u\|_{\mathbf{F}^{1}(T)}^{2} \\
\|u\|_{\mathbf{B}^{1}(T)}^{2} \lesssim\|\phi\|_{\mathbf{E}^{1}}^{2}+\|u\|_{\mathbf{F}^{1}(T)}^{3}
\end{array}\right.
$$

The inequalities (1.12) and a simple continuity argument still suffice to control $\|u\|_{\mathbf{F}^{1}(T)}$, provided that $\|\phi\|_{\mathbf{E}^{1}} \ll 1$ (which can be arranged by rescaling). The first inequality in (1.12) is the analogue of the linear estimate (1.10), and uses the linear equation (1.9). The second inequality in (1.12) is the analogue of the bilinear estimate (1.11). The last inequality in (1.12) is an energy-type estimate.

To prove Theorem 1.1 (b) we need to exploit several special symmetries of the equation satisfied by the difference of two solutions. This difference equation has special symmetries for real-valued solutions in $L^{2}$ and in $\dot{H}_{x}^{-1}$. To exploit these symmetries, we define the normed spaces $\overline{\mathbf{F}}^{0}, \overline{\mathbf{N}}^{0}$, and the semi-normed space $\overline{\mathbf{B}}^{0}$, and prove a second set of linear, bilinear, and energy estimates, similar to (1.12). Then we adapt the Bona-Smith method [2] to prove the continuity of the flow in the space $\mathbf{E}^{1}$.

We explain now our strategy to define the main normed and semi-normed spaces. Ideally, one would like to use standard $X^{s, b}$ - type structures (as in [3], [10]) for the spaces $\mathbf{F}^{1}(T)$ and $\mathbf{N}^{1}(T)$. For such spaces, however, the bilinear estimate $\left\|\partial_{x}(u v)\right\|_{\mathbf{N}^{1}(T)} \lesssim\|u\|_{\mathbf{F}^{1}(T)}\|v\|_{\mathbf{F}^{1}(T)}$ cannot hold even for solutions $u, v$ of the linear homogeneous equation. This bilinear estimate is only possible if we weaken significantly the contributions of the components of the functions $u$ and $v$ of high frequency and low modulation. To achieve this we still use $X^{s, b}$ type structures for the spaces $\mathbf{F}^{1}(T)$ and $\mathbf{N}^{1}(T)$, but only on small, frequency dependent time intervals. A similar method was used recently in [5] and [13] to prove a-priori bounds for the 1-d cubic nonlinear Schrödinger equation in negative Sobolev spaces.

The second step is to define $\|u\|_{\mathbf{B}^{1}(T)}$ sufficiently large to be able to still prove the linear estimate $\|u\|_{\mathbf{F}^{1}(T)} \lesssim\|u\|_{\mathbf{B}^{1}(T)}+\left\|-\partial_{x}\left(u^{2} / 2\right)\right\|_{\mathbf{N}^{1}(T)}$. Finally, we use frequency-localized energy estimates and the symmetries of the equation (1.1) $]^{2}$ to prove the energy estimate $\|u\|_{\mathbf{B}^{1}(T)}^{2} \lesssim\|\phi\|_{\mathbf{E}^{1}}^{2}+\|u\|_{\mathbf{F}^{1}(T)}^{3}$. These symmetries allow us to trade high frequencies for low frequencies in trilinear forms, improving the

\footnotetext{
${ }^{2}$ The two main symmetries used at this stage are the fact that the solution $u$ is real-valued and the precise form of the nonlinearity $-\partial_{x}\left(u^{2} / 2\right)$.
} 
timescale from frequency dependent time intervals (as guaranteed by the bilinear estimates) to frequency independent time intervals.

A new twist arises in the proof of part (b) of Theorem 1.1. The symmetries of the difference equation are not as good as the symmetries of the nonlinear equation, which causes difficulties in the proofs of suitable energy estimates. The low frequency part of the solution turns out to be particularly harmful in the difference equation. To avoid this difficulty we define the normed spaces $\overline{\mathbf{F}}^{0}, \overline{\mathbf{N}}^{0}$, and the semi-normed space $\overline{\mathbf{B}}^{0}$, which have a special low-frequency structure.

The rest of the paper is organized as follows: in section 2 we summarize most of the notation, define the main normed spaces, and prove some of their basic properties. In section 3 we state our main global linear, bilinear, and energy estimates. The proof of the bilinear estimate Proposition 3.3 depends on the dyadic bilinear estimates proved in sections 7 and 8; the energy estimates Proposition 3.4 and Proposition 3.6 are proved in section 6 . In section 4 we prove the main theorem, using the linear, bilinear, and energy estimates of section 3 . In section 5, which is self-contained, we prove the bilinear $L^{2}$ estimates in Corollary 5.3. these $L^{2}$ estimates are the main building blocks in all the dyadic estimates in sections 6, 7, and 8, The key technical ingredient is the scale-invariant estimate in Lemma 5.1 (a), which is also used in [6]. In section 6] we prove the energy estimates Proposition 3.4 and Proposition 3.6. Finally, in sections 7 and 8 we prove the dyadic bilinear estimates used in Proposition 3.3 .

\section{Notation AND DEFinitions}

Let $\mathbb{Z}_{+}=\mathbb{Z} \cap[0, \infty)$. For $k \in \mathbb{Z}$ let

$$
I_{k}=\left\{\xi:|\xi| \in\left[(3 / 4) \cdot 2^{k},(3 / 2) \cdot 2^{k}\right]\right\} \text { and } \widetilde{I}_{k}=\left\{\xi:|\xi| \in\left[2^{k-1}, 2^{k+1}\right]\right\} .
$$

Let $\eta_{0}: \mathbb{R} \rightarrow[0,1]$ denote an even smooth function supported in $[-8 / 5,8 / 5]$ and equal to 1 in $[-5 / 4,5 / 4]$. For $k \in \mathbb{Z} \cap[1, \infty)$ let $\eta_{k}(\xi)=\eta_{0}\left(\xi / 2^{k}\right)-\eta_{0}\left(\xi / 2^{k-1}\right)$. For $k \in \mathbb{Z}_{+}$let $\eta_{\leq k}=\sum_{k^{\prime}=0}^{k} \eta_{k^{\prime}}$. For $k \in \mathbb{Z}$ let $\chi_{k}(\xi)=\eta_{0}\left(\xi / 2^{k}\right)-\eta_{0}\left(\xi / 2^{k-1}\right)$. For $(\xi, \mu) \in \mathbb{R} \backslash\{0\} \times \mathbb{R}$ let

$$
\omega(\xi, \mu)=\xi^{3}+\mu^{2} / \xi
$$

For $k \in \mathbb{Z}$ we define the dyadic $X^{s, b}$-type normed spaces $X_{k}=X_{k}\left(\mathbb{R}^{3}\right)$,

$$
\begin{aligned}
& X_{k}=\left\{f \in L^{2}\left(\mathbb{R}^{3}\right): f \text { is supported in } \widetilde{I}_{k} \times \mathbb{R} \times \mathbb{R}\right. \text { and } \\
& \left.\qquad\|f\|_{X_{k}}=\sum_{j=0}^{\infty} 2^{j / 2}\left\|\eta_{j}(\tau-\omega(\xi, \mu)) \cdot f\right\|_{L^{2}}<\infty\right\} .
\end{aligned}
$$

We use an $l^{1}$ Besov-type norm with respect to modulations. Structures of this type were introduced, for instance, in [19], and are useful in order to prevent high frequency losses in bilinear and trilinear estimates. 
The definition shows easily that if $k \in \mathbb{Z}$ and $f_{k} \in X_{k}$ then

$$
\left\|\int_{\mathbb{R}}\left|f_{k}\left(\xi, \mu, \tau^{\prime}\right)\right| d \tau^{\prime}\right\|_{L_{\xi, \mu}^{2}} \lesssim\left\|f_{k}\right\|_{X_{k}} .
$$

Moreover, if $k \in \mathbb{Z}, l \in \mathbb{Z}_{+}$, and $f_{k} \in X_{k}$ then

$$
\begin{aligned}
& \sum_{j=l+1}^{\infty} 2^{j / 2}\left\|\eta_{j}(\tau-\omega(\xi, \mu)) \cdot \int_{\mathbb{R}}\left|f_{k}\left(\xi, \mu, \tau^{\prime}\right)\right| \cdot 2^{-l}\left(1+2^{-l}\left|\tau-\tau^{\prime}\right|\right)^{-4} d \tau^{\prime}\right\|_{L^{2}} \\
& +2^{l / 2}\left\|\eta_{\leq l}(\tau-\omega(\xi, \mu)) \cdot \int_{\mathbb{R}}\left|f_{k}\left(\xi, \mu, \tau^{\prime}\right)\right| \cdot 2^{-l}\left(1+2^{-l}\left|\tau-\tau^{\prime}\right|\right)^{-4} d \tau^{\prime}\right\|_{L^{2}} \\
& \lesssim\left\|f_{k}\right\|_{X_{k}} .
\end{aligned}
$$

In particular, if $k \in \mathbb{Z}, l \in \mathbb{Z}_{+}, t_{0} \in \mathbb{R}, f_{k} \in X_{k}$, and $\gamma \in \mathcal{S}(\mathbb{R})$, then

$$
\left\|\mathcal{F}\left[\gamma\left(2^{l}\left(t-t_{0}\right)\right) \cdot \mathcal{F}^{-1}\left(f_{k}\right)\right]\right\|_{X_{k}} \lesssim\left\|f_{k}\right\|_{X_{k}} .
$$

For $k \in \mathbb{Z}$ let $k_{+}=\max (k, 0)$, and let $P_{k}$ denote the operator on $L^{2}\left(\mathbb{R}^{3}\right)$ defined by the Fourier multiplier $(\xi, \mu, \tau) \rightarrow \mathbf{1}_{I_{k}}(\xi)$. By a slight abuse of notation, we also let $P_{k}$ denote the operator on $L^{2}\left(\mathbb{R}^{2}\right)$ defined by the Fourier multiplier $(\xi, \mu) \rightarrow \mathbf{1}_{I_{k}}(\xi)$. For $l \in \mathbb{Z}$ let

$$
P_{\leq l}=\sum_{k \leq l} P_{k}, \quad P_{\geq l}=\sum_{k \geq l} P_{k}
$$

With $p$ as in (1.3), for $k \in \mathbb{Z}$ define the frequency localized initial data spaces

$$
E_{k}=\left\{\phi: \mathbb{R}^{2} \rightarrow \mathbb{R}: \mathcal{F}(\phi)=\mathbf{1}_{\widetilde{I}_{k}}(\xi) \mathcal{F}(\phi) \text { and }\|\phi\|_{E_{k}}=\|\widehat{\phi} \cdot p(\xi, \mu)\|_{L_{\xi, \mu}^{2}}<\infty\right\}
$$

and

$$
\bar{E}_{k}=\left\{\phi: \mathbb{R}^{2} \rightarrow \mathbb{R}: \mathcal{F}(\phi)=\mathbf{1}_{\widetilde{I}_{k}}(\xi) \mathcal{F}(\phi) \text { and }\|\phi\|_{\bar{E}_{k}}=\|\widehat{\phi}\|_{L_{\xi, \mu}^{2}}<\infty\right\} .
$$

The corresponding frequency localized energy spaces for the solutions are

$$
\begin{aligned}
& C_{0}\left(\mathbb{R}: E_{k}\right)=\left\{u_{k} \in C\left(\mathbb{R}: E_{k}\right): u_{k} \text { is supported in } \mathbb{R}^{2} \times[-4,4]\right\} \\
& C_{0}\left(\mathbb{R}: \bar{E}_{k}\right)=\left\{u_{k} \in C\left(\mathbb{R}: \bar{E}_{k}\right): u_{k} \text { is supported in } \mathbb{R}^{2} \times[-4,4]\right\} .
\end{aligned}
$$

At frequency $2^{k}$ we will use the $X^{s, b}$ structure given by the $X_{k}$ norm, uniformly on the $2^{-k_{+}}$time scale. For $k \in \mathbb{Z}$ we define the normed spaces

$$
F_{k}=\left\{u_{k} \in C_{0}\left(\mathbb{R}: E_{k}\right):\left\|u_{k}\right\|_{F_{k}}=\sup _{t_{k} \in \mathbb{R}}\left\|p(\xi, \mu) \cdot \mathcal{F}\left[u_{k} \cdot \eta_{0}\left(2^{k_{+}}\left(t-t_{k}\right)\right)\right]\right\|_{X_{k}}<\infty\right\},
$$

and

$$
\bar{F}_{k}=\left\{u_{k} \in C_{0}\left(\mathbb{R}: \bar{E}_{k}\right):\left\|u_{k}\right\|_{\bar{F}_{k}}=\sup _{t_{k} \in \mathbb{R}}\left\|\mathcal{F}\left[u_{k} \cdot \eta_{0}\left(2^{k_{+}}\left(t-t_{k}\right)\right)\right]\right\|_{X_{k}}<\infty\right\}
$$


For $k \in \mathbb{Z}$ we define the normed spaces $N_{k}=C_{0}\left(\mathbb{R}: E_{k}\right)$ and $\bar{N}_{k}=C_{0}\left(\mathbb{R}: \bar{E}_{k}\right)$ (as vector spaces), which are used to measure the frequency $2^{k}$ part of the nonlinear term, with norms

$$
\left\|f_{k}\right\|_{N_{k}}=\sup _{t_{k} \in \mathbb{R}}\left\|p(\xi, \mu)\left(\tau-\omega(\xi, \mu)+i 2^{k_{+}}\right)^{-1} \cdot \mathcal{F}\left[f_{k} \cdot \eta_{0}\left(2^{k_{+}}\left(t-t_{k}\right)\right)\right]\right\|_{X_{k}},
$$

and

$$
\left\|u_{k}\right\|_{\bar{N}_{k}}=\sup _{t_{k} \in \mathbb{R}}\left\|\left(\tau-\omega(\xi, \mu)+i 2^{k_{+}}\right)^{-1} \cdot \mathcal{F}\left[f_{k} \cdot \eta_{0}\left(2^{k_{+}}\left(t-t_{k}\right)\right)\right]\right\|_{X_{k}} .
$$

The bounds we obtain for solutions of the KP-I equation are on a fixed time interval, while the above function spaces are not. To remedy this, for any time $T \in(0,1]$ we define the normed spaces

$$
\left\{\begin{array}{l}
F_{k}(T)=\left\{u_{k} \in C\left([-T, T]: E_{k}\right):\left\|u_{k}\right\|_{F_{k}(T)}=\underset{\widetilde{u}_{k}=u_{k} \operatorname{in} \mathbb{R}^{2} \times[-T, T]}{\inf _{\tilde{f}_{k}=f_{k}} \operatorname{in} \mathbb{R}^{2} \times[-T, T]}\left\|\widetilde{u}_{k}\right\|_{F_{k}}<\infty\right\} ; \\
N_{k}(T)=\left\{f_{k} \in C\left([-T, T]: E_{k}\right):\left\|u_{k}\right\|_{N_{k}(T)}=\infty\right\},
\end{array}\right.
$$

where the infimum is taken over all extensions $\widetilde{u}_{k} \in C_{0}\left(\mathbb{R}: E_{k}\right)$ of $u_{k}$. Similarly we define the normed spaces

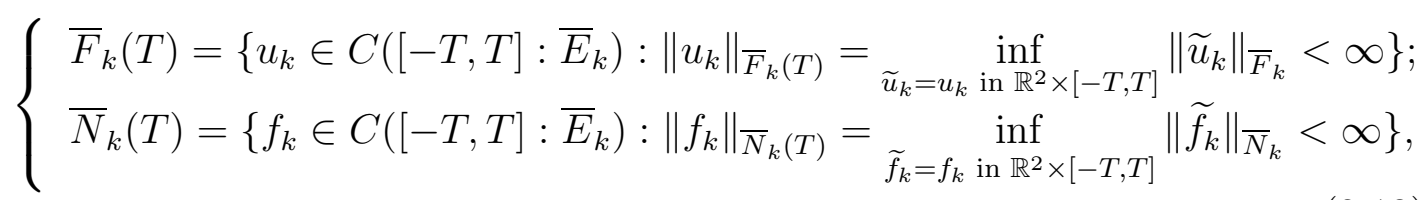

where the infimum is taken over all extensions $\widetilde{u}_{k} \in C_{0}\left(\mathbb{R}: \bar{E}_{k}\right)$ of $u_{k}$.

So far we have defined the dyadic function spaces where we measure the solution $u$ to (1.1) and the nonlinearity. We assemble these in a straightforward manner using a Littlewood-Paley decomposition to obtain the global function spaces for the solutions. In what follows we let $\sigma \in \mathbb{Z}_{+}$and $T \in(0,1]$. We define the Banach spaces for the initial data

$$
\mathbf{E}^{\sigma}=\left\{\phi: \mathbb{R}^{2} \rightarrow \mathbb{R}:\|\phi\|_{\mathbf{E}^{\sigma}}=\left\|\widehat{\phi} \cdot p(\xi, \mu)(1+|\xi|)^{\sigma}\right\|_{L_{\xi, \mu}^{2}}<\infty\right\},
$$

and

$$
\overline{\mathbf{E}}^{\sigma}=\left\{\phi: \mathbb{R}^{2} \rightarrow \mathbb{R}:\|\phi\|_{\overline{\mathbf{E}}^{\sigma}}=\left\|\widehat{\phi} \cdot\left(1+|\xi|^{-5 / 8}\right)(1+|\xi|)^{\sigma}\right\|_{L_{\xi, \mu}^{2}}<\infty\right\} .
$$

Their intersections are denoted by

$$
\mathbf{E}^{\infty}=\bigcap_{\sigma=1}^{\infty} \mathbf{E}^{\sigma}, \quad \overline{\mathbf{E}}^{\infty}=\bigcap_{\sigma=1}^{\infty} \overline{\mathbf{E}}^{\sigma}
$$

For $u \in C\left([-T, T]: \mathbf{E}^{\infty}\right)$, respectively $u \in C\left([-T, T]: \overline{\mathbf{E}}^{\infty}\right)$, we define

$$
\|u\|_{\mathbf{B}^{\sigma}(T)}^{2}=\left\|P_{\leq 0}(u(0))\right\|_{\mathbf{E}^{\sigma}}^{2}+\sum_{k \geq 1} \sup _{t_{k} \in[-T, T]} 2^{2 \sigma k}\left\|P_{k}\left(u\left(t_{k}\right)\right)\right\|_{E_{k}}^{2},
$$


and

$$
\|u\|_{\overline{\mathbf{B}}^{\sigma}(T)}^{2}=\left\|P_{\leq 0}(u(0))\right\|_{\overline{\mathbf{E}}^{0}}^{2}+\sum_{k \geq 1} \sup _{t_{k} \in[-T, T]} 2^{2 \sigma k}\left\|P_{k}\left(u\left(t_{k}\right)\right)\right\|_{\bar{E}_{k}}^{2} .
$$

Notice that the $\overline{\mathbf{E}}^{\sigma}$ and $\overline{\mathbf{B}}^{\sigma}(T)$ norms, which are used for the difference equation, have the added factor $\left(1+|\xi|^{-5 / 8}\right)$. This gives extra decay at low frequencies, and is essential in our analysis.

Finally, the $X^{s, b}$ - type control of the solutions, respectively the nonlinearity is achieved using the normed spaces

$$
\begin{aligned}
& \mathbf{F}^{\sigma}(T)=\left\{u \in C\left([-T, T]: \mathbf{E}^{\infty}\right):\|u\|_{\mathbf{F}^{\sigma}(T)}^{2}=\sum_{k \in \mathbb{Z}} 2^{2 \sigma k_{+}}\left\|P_{k}(u)\right\|_{F_{k}(T)}^{2}<\infty\right\}, \\
& \mathbf{N}^{\sigma}(T)=\left\{u \in C\left([-T, T]: \mathbf{E}^{\infty}\right):\|u\|_{\mathbf{N}^{\sigma}(T)}^{2}=\sum_{k \in \mathbb{Z}} 2^{2 \sigma k_{+}}\left\|P_{k}(u)\right\|_{N_{k}(T)}^{2}<\infty\right\} .
\end{aligned}
$$

For the difference equation we use the normed spaces

$$
\begin{aligned}
& \overline{\mathbf{F}}^{0}(T)=\left\{u \in C\left([-T, T]: \overline{\mathbf{E}}^{\infty}\right):\|u\|_{\overline{\mathbf{F}}^{0}(T)}^{2}=\sum_{k \in \mathbb{Z}}\left(1+2^{-5 k / 4}\right)\left\|P_{k}(u)\right\|_{\bar{F}_{k}(T)}^{2}<\infty\right\}, \\
& \overline{\mathbf{N}}^{0}(T)=\left\{u \in C\left([-T, T]: \overline{\mathbf{E}}^{\infty}\right):\|u\|_{\overline{\mathbf{N}}^{0}(T)}^{2}=\sum_{k \in \mathbb{Z}}\left(1+2^{-5 k / 4}\right)\left\|P_{k}(u)\right\|_{\bar{N}_{k}(T)}^{2}<\infty\right\} .
\end{aligned}
$$

For any $k \in \mathbb{Z}$ we define the set $S_{k}$ of $k$-acceptable time multiplication factors

$$
S_{k}=\left\{m_{k}: \mathbb{R} \rightarrow \mathbb{R}:\left\|m_{k}\right\|_{S_{k}}=\sum_{j=0}^{10} 2^{-j k_{+}}\left\|\partial^{j} m_{k}\right\|_{L^{\infty}}<\infty\right\} .
$$

Direct estimates using the definitions and (2.4) show that for any $\sigma \in \mathbb{Z}_{+}$and $T \in(0,1]$

$$
\left\{\begin{aligned}
\left\|\sum_{k \in \mathbb{Z}} m_{k}(t) \cdot P_{k}(u)\right\|_{\mathbf{F}^{\sigma}(T)} & \lesssim\left(\sup _{k \in \mathbb{Z}}\left\|m_{k}\right\|_{S_{k}}\right) \cdot\|u\|_{\mathbf{F}^{\sigma}(T)} \\
\left\|\sum_{k \in \mathbb{Z}} m_{k}(t) \cdot P_{k}(u)\right\|_{\mathbf{N}^{\sigma}(T)} & \lesssim\left(\sup _{k \in \mathbb{Z}}\left\|m_{k}\right\|_{S_{k}}\right) \cdot\|u\|_{\mathbf{N}^{\sigma}(T)} \\
\left\|\sum_{k \in \mathbb{Z}} m_{k}(t) \cdot P_{k}(u)\right\|_{\mathbf{B}^{\sigma}(T)} & \lesssim\left(\sup _{k \in \mathbb{Z}}\left\|m_{k}\right\|_{S_{k}}\right) \cdot\|u\|_{\mathbf{B}^{\sigma}(T)}
\end{aligned}\right.
$$

and

$$
\left\{\begin{aligned}
\left\|\sum_{k \in \mathbb{Z}} m_{k}(t) \cdot P_{k}(u)\right\|_{\overline{\mathbf{F}}^{0}(T)} & \lesssim\left(\sup _{k \in \mathbb{Z}}\left\|m_{k}\right\|_{S_{k}}\right) \cdot\|u\|_{\overline{\mathbf{F}}^{0}(T)} \\
\left\|\sum_{k \in \mathbb{Z}} m_{k}(t) \cdot P_{k}(u)\right\|_{\overline{\mathbf{N}}^{0}(T)} \lesssim\left(\sup _{k \in \mathbb{Z}}\left\|m_{k}\right\|_{S_{k}}\right) \cdot\|u\|_{\overline{\mathbf{N}}^{0}(T)} & \lesssim\left(\sup _{k \in \mathbb{Z}}\left\|m_{k}\right\|_{S_{k}}\right) \cdot\|u\|_{\overline{\mathbf{B}}^{0}(T)}
\end{aligned}\right.
$$




\section{Global linear, Bilinear And energy estimates}

In this section we state our main linear, bilinear and energy estimates. We show first that $\mathbf{F}^{\sigma}(T) \hookrightarrow C\left([-T, T]: \mathbf{E}^{\sigma}\right)$ for $\sigma \in \mathbb{Z}_{+}, T \in(0,1]$.

Lemma 3.1. If $\sigma \in \mathbb{Z}_{+}, T \in(0,1]$, and $u \in \mathbf{F}^{\sigma}(T)$, then

$$
\sup _{t \in[-T, T]}\|u(t)\|_{\mathbf{E}^{\sigma}} \lesssim\|u\|_{\mathbf{F}^{\sigma}(T)} .
$$

Proof of Lemma 3.1. In view of the definitions, it suffices to prove that if $k \in \mathbb{Z}$, $t_{k} \in[-1,1]$, and $\widetilde{u}_{k} \in F_{k}$ then

$$
\left\|p(\xi, \mu) \cdot \mathcal{F}\left[\widetilde{u}_{k}\left(t_{k}\right)\right]\right\|_{L_{\xi, \mu}^{2}} \lesssim\left\|p(\xi, \mu) \cdot \mathcal{F}\left[\widetilde{u}_{k} \cdot \eta_{0}\left(2^{k_{+}}\left(t-t_{k}\right)\right)\right]\right\|_{X_{k}} .
$$

Let $f_{k}=\mathcal{F}\left[\widetilde{u}_{k} \cdot \eta_{0}\left(2^{k_{+}}\left(t-t_{k}\right)\right)\right]$, so

$$
\mathcal{F}\left[\widetilde{u}_{k}\left(t_{k}\right)\right](\xi, \mu)=c \int_{\mathbb{R}} f_{k}(\xi, \mu, \tau) e^{i t_{k} \tau} d \tau .
$$

Thus, using (2.3),

$$
\left\|p(\xi, \mu) \cdot \mathcal{F}\left[\widetilde{u}_{k}\left(t_{k}\right)\right]\right\|_{L_{\xi, \mu}^{2}} \lesssim\left\|p(\xi, \mu) \cdot f_{k}\right\|_{L_{\xi, \mu}^{2} L_{\tau}^{1}} \lesssim\left\|p(\xi, \mu) \cdot f_{k}\right\|_{X_{k}},
$$

which gives (3.2).

We prove now a linear estimate.

Proposition 3.2. Assume $T \in(0,1], u, v \in C\left([-T, T): \mathbf{E}^{\infty}\right)$ and

$$
\partial_{t} u+\partial_{x}^{3} u-\partial_{x}^{-1} \partial_{y}^{2} u=v \text { on } \mathbb{R}^{2} \times(-T, T) .
$$

(a) Then, for any $\sigma \in \mathbb{Z}_{+}$,

$$
\|u\|_{\mathbf{F}^{\sigma}(T)} \lesssim\|u\|_{\mathbf{B}^{\sigma}(T)}+\|v\|_{\mathbf{N}^{\sigma}(T)} .
$$

(b) Assume, in addition, that $u(0) \in \overline{\mathbf{E}}^{\infty}$ and $v \in \overline{\mathbf{N}}^{0}(T)$. Then $u \in \overline{\mathbf{F}}^{0}(T)$ and

$$
\|u\|_{\overline{\mathbf{F}}^{0}(T)} \lesssim\|u\|_{\overline{\mathbf{B}}^{0}(T)}+\|v\|_{\overline{\mathbf{N}}^{0}(T)}
$$

Proof of Proposition [3.2. In view of the definitions, it suffices to prove that if $k \in \mathbb{Z}$ and $u, v \in C\left([-T, T]: E_{k}\right)$ solve $(3.3)$, then

$$
\left\{\begin{aligned}
\left\|P_{k}(u)\right\|_{F_{k}(T)} & \lesssim\left\|P_{k}(u(0))\right\|_{E_{k}}+\|v\|_{N_{k}(T)} \text { if } k \leq 0 \\
\left\|P_{k}(u)\right\|_{F_{k}(T)} & \lesssim \sup _{t_{k} \in[-T, T]}\left\|P_{k}\left(u\left(t_{k}\right)\right)\right\|_{E_{k}}+\|v\|_{N_{k}(T)} \text { if } k \geq 1 .
\end{aligned}\right.
$$

Let $\widetilde{v} \in C_{0}\left(\mathbb{R}: E_{k}\right)$ denote an extension of $v$ such that $\|\widetilde{v}\|_{N_{k}} \leq C\|v\|_{N_{k}(T)}$. Using (2.21), we may assume that $\widetilde{v}$ is supported in $\mathbb{R}^{2} \times\left[-T-2^{-k_{+}-10}, T+2^{-k_{+}-10}\right]$, $k \in \mathbb{Z}$. For $t \in \mathbb{R}$ let $W(t)$ denote the solution at time $t$ of the free KP-I evolution, 
i.e. the operator on $L^{2}\left(\mathbb{R}^{2}\right)$ defined by the Fourier multiplier $(\xi, \mu) \rightarrow e^{i t \omega(\xi, \mu)}$. For $t \geq T$ we define

$$
\widetilde{u}(t)=\eta_{0}\left(2^{k_{+}+5}(t-T)\right)\left[W(t-T) P_{k}(u(T))+\int_{T}^{t} W(t-s)\left(P_{k}(\widetilde{v}(s))\right) d s\right] .
$$

For $t \leq-T$ we define

$$
\widetilde{u}(t)=\eta_{0}\left(2^{k_{+}+5}(t+T)\right)\left[W(t+T) P_{k}(u(-T))+\int_{-T}^{t} W(t-s)\left(P_{k}(\widetilde{v}(s))\right) d s\right] .
$$

With $\widetilde{u}(t)=u(t)$ for $t \in[-T, T]$, it is clear that $\widetilde{u} \in C_{0}\left(\mathbb{R}: E_{k}\right)$ is an extension of $u$. Also, using (2.21),

$$
\|u\|_{F_{k}(T)} \lesssim \sup _{t_{k} \in[-T, T]}\left\|p(\xi, \mu) \cdot \mathcal{F}\left[\widetilde{u} \cdot \eta_{0}\left(2^{k_{+}}\left(t-t_{k}\right)\right)\right]\right\|_{X_{k}}
$$

where the supremum is taken over $t_{k} \in[-T, T]$.

In view of the definitions and (2.21), it suffices to prove that if $k \in \mathbb{Z}, \phi_{k} \in E_{k}$, and $v_{k} \in N_{k}$ then

$$
\begin{aligned}
\left\|p(\xi, \mu) \cdot \mathcal{F}\left[u_{k} \cdot \eta_{0}\left(2^{k_{+}} t\right)\right]\right\|_{X_{k}} \lesssim\left\|p(\xi, \mu) \cdot \widehat{\phi_{k}}\right\|_{L_{\xi, \mu}^{2}} \\
+\left\|p(\xi, \mu)\left(\tau-\omega(\xi, \mu)+i 2^{k_{+}}\right)^{-1} \cdot \mathcal{F}\left(v_{k}\right)\right\|_{X_{k}}
\end{aligned}
$$

where

$$
u_{k}(t)=W(t)\left(\phi_{k}\right)+\int_{0}^{t} W(t-s)\left(v_{k}(s)\right) d s .
$$

It follows from (3.8) that

$$
\begin{aligned}
\mathcal{F} & {\left[u_{k} \cdot \eta_{0}\left(2^{k_{+}} t\right)\right](\xi, \mu, \tau)=\widehat{\phi}_{k}(\xi, \mu) \cdot 2^{-k_{+}} \widehat{\eta}_{0}\left(2^{-k_{+}}(\tau-\omega(\xi, \mu))\right) } \\
& +C \int_{\mathbb{R}} \mathcal{F}\left(v_{k}\right)\left(\xi, \mu, \tau^{\prime}\right) \cdot \frac{2^{-k_{+}} \widehat{\eta}_{0}\left(2^{-k_{+}}\left(\tau-\tau^{\prime}\right)\right)-2^{-k_{+}} \widehat{\eta}_{0}\left(2^{-k_{+}}(\tau-\omega(\xi, \mu))\right)}{\tau^{\prime}-\omega(\xi, \mu)} d \tau^{\prime} .
\end{aligned}
$$

We observe now that

$$
\begin{aligned}
& \left|\frac{2^{-k_{+}} \widehat{\eta}_{0}\left(2^{-k_{+}}\left(\tau-\tau^{\prime}\right)\right)-2^{-k_{+}} \widehat{\eta}_{0}\left(2^{-k_{+}}(\tau-\omega(\xi, \mu))\right)}{\tau^{\prime}-\omega(\xi, \mu)} \cdot\left(\tau-\omega(\xi, \mu)+i 2^{k_{+}}\right)\right| \\
& \quad \lesssim 2^{-k_{+}}\left(1+2^{-k_{+}}\left|\tau-\tau^{\prime}\right|\right)^{-4}+2^{-k_{+}}\left(1+2^{-k_{+}}|\tau-\omega(\xi, \mu)|\right)^{-4},
\end{aligned}
$$

and the bound (3.7) follows from (2.3) and (2.4).

We continue with our main bilinear estimates.

Proposition 3.3. a) If $\sigma \in\{1,2,3\}, T \in[0,1)$, and $u, v \in \mathbf{F}^{\sigma}(T)$ then

$$
\left\|\partial_{x}(u v)\right\|_{\mathbf{N}^{\sigma}(T)} \lesssim\|u\|_{\mathbf{F}^{\sigma}(T)} \cdot\|v\|_{\mathbf{F}^{1}(T)}+\|u\|_{\mathbf{F}^{1}(T)} \cdot\|v\|_{\mathbf{F}^{\sigma}(T)} .
$$

b) If $T \in(0,1], u \in \overline{\mathbf{F}}^{0}(T)$ and $v \in \mathbf{F}^{1}(T)$ then

$$
\left\|\partial_{x}(u v)\right\|_{\overline{\mathbf{N}}^{0}(T)} \lesssim\|u\|_{\overline{\mathbf{F}}^{0}(T)} \cdot\|v\|_{\mathbf{F}^{1}(T)} \cdot
$$


Proof of Proposition [3.3. We fix extensions $\widetilde{u}, \widetilde{v} \in C_{0}\left(\mathbb{R}: \mathbf{E}^{\infty}\right)$ of $u, v$ such that $\left\|P_{k}(\widetilde{u})\right\|_{F_{k}} \leq 2\left\|P_{k}(u)\right\|_{F_{k}(T)}$ and $\left\|P_{k}(\widetilde{v})\right\|_{F_{k}} \leq 2\left\|P_{k}(v)\right\|_{F_{k}(T)}, k \in \mathbb{Z}$. Let $\widetilde{u}_{k}=$ $P_{k}(\widetilde{u})$ and $\widetilde{v}_{k}=P_{k}(\widetilde{v}), k \in \mathbb{Z}$. It follows from Lemma 7.1, Lemma 7.2, and Lemma 7.5 that

$$
\left\{\begin{array}{l}
2^{k_{+}}\left\|P_{k}\left(\partial_{x}\left(\widetilde{u}_{k_{1}} \widetilde{v}_{k_{2}}\right)\right)\right\|_{N_{k}} \lesssim 2^{-\left|k_{1}\right| / 2} 2^{k_{1+}}\left\|\widetilde{u}_{k_{1}}\right\|_{F_{k_{1}}} \cdot 2^{k_{2+}}\left\|\widetilde{v}_{k_{2}}\right\|_{F_{k_{2}}} \\
\text { if } k_{1} \leq k_{2} \text { and }\left|k_{2}-k\right| \leq 40 .
\end{array}\right.
$$

Also, it follows from Lemma 7.3, Lemma 7.4, and Lemma 7.5 that

$$
\left\{\begin{array}{l}
2^{k_{+}}\left\|P_{k}\left(\partial_{x}\left(\widetilde{u}_{k_{1}} \widetilde{v}_{k_{2}}\right)\right)\right\|_{N_{k}} \lesssim 2^{-\max \left(|k|,\left|k_{1}\right|,\left|k_{2}\right|\right) / 4} \cdot 2^{k_{1+}}\left\|\widetilde{u}_{k_{1}}\right\|_{F_{k_{1}}} \cdot 2^{k_{2+}}\left\|\widetilde{v}_{k_{2}}\right\|_{F_{k_{2}}} \\
\text { if }\left|k_{1}-k_{2}\right| \leq 4 \text { and } k \leq \min \left(k_{1}, k_{2}\right)-30 .
\end{array}\right.
$$

The bound (3.9) follows from (3.11) and (3.12).

Consider now part (b) of the proposition. We fix extensions $\widetilde{u} \in C_{0}\left(\mathbb{R}: \overline{\mathbf{E}}^{\infty}\right)$ of $u$ and $\widetilde{v} \in C\left(\mathbb{R}: \mathbf{E}^{\infty}\right)$ of $v$ such that $\left\|P_{k}(\widetilde{u})\right\|_{F_{k}} \leq 2\left\|P_{k}(u)\right\|_{F_{k}(T)}$ and $\left\|P_{k}(\widetilde{v})\right\|_{F_{k}} \leq$ $2\left\|P_{k}(v)\right\|_{F_{k}(T)}, k \in \mathbb{Z}$. Let $\widetilde{u}_{k}=P_{k}(\widetilde{u})$ and $\widetilde{v}_{k}=P_{k}(\widetilde{v}), k \in \mathbb{Z}$. It follows from Lemma 8.1, Lemma 8.2, and Lemma 8.4 that

$$
\begin{aligned}
&\left(1+2^{-5 k / 8}\right)\left\|P_{k}\left(\partial_{x}\left(\widetilde{u}_{k_{1}} \widetilde{v}_{k_{2}}\right)\right)\right\|_{\bar{N}_{k}} \lesssim 2^{-\max \left(|k|,\left|k_{1}\right|,\left|k_{2}\right|\right) / 8} \\
&\left(1+2^{-5 k_{1} / 8}\right)\left\|\widetilde{u}_{k_{1}}\right\|_{\bar{F}_{k_{1}}} \cdot\left(1+2^{k_{2}}\right)\left\|v_{k_{2}}\right\|_{F_{k_{2}}}
\end{aligned}
$$

if $\left|k_{1}-k\right| \geq 5$. It follows from Lemma 8.1. Lemma 8.2, and Lemma 8.3 that

$$
\left(1+2^{-5 k / 8}\right)\left\|P_{k}\left(\partial_{x}\left(\widetilde{u}_{k_{1}} \widetilde{v}_{k_{2}}\right)\right)\right\|_{\bar{N}_{k}} \lesssim 2^{-\left|k_{2}\right| / 8}\left(1+2^{-5 k_{1} / 8}\right)\left\|\widetilde{u}_{k_{1}}\right\|_{\bar{F}_{k_{1}}} \cdot\left(1+2^{k_{2}}\right)\left\|v_{k_{2}}\right\|_{F_{k_{2}}}
$$

if $\left|k_{1}-k\right| \leq 4$. The proposition follows.

The last ingredients in the proof of Theorem 1.1 are energy estimates. For part (a) we need the following:

Proposition 3.4. Assume that $T \in(0,1]$ and $u \in C\left([-T, T]: \mathbf{E}^{\infty}\right)$ is a solution of the initial value problem

$$
\left\{\begin{array}{l}
\partial_{t} u+\partial_{x}^{3} u-\partial_{x}^{-1} \partial_{y}^{2} u+\partial_{x}\left(u^{2} / 2\right)=0 \text { on } \mathbb{R}^{2} \times[-T, T] \\
u(0)=\phi
\end{array}\right.
$$

Then, for $\sigma \in\{1,2,3\}$ we have

$$
\|u\|_{\mathbf{B}^{\sigma}(T)}^{2} \lesssim\|\phi\|_{\mathbf{E}^{\sigma}}^{2}+\|u\|_{\mathbf{F}^{1}(T)} \cdot\|u\|_{\mathbf{F}^{\sigma}(T)}^{2} .
$$

The linearized equation lacks the full set of symmetries of the nonlinear equation. Consequently, we have good estimates for it only at the $L^{2}$ level:

Proposition 3.5. Assume $T \in(0,1], u \in \overline{\mathbf{F}}^{0}(T) \cap \mathbf{F}^{1}(T), v \in \mathbf{F}^{1}(T)$ and

$$
\left\{\begin{array}{l}
\partial_{t} u+\partial_{x}^{3} u-\partial_{x}^{-1} \partial_{y}^{2} u+\partial_{x}(u v)=0 \text { on } \mathbb{R}^{2} \times(-T, T) \\
u(0)=\phi
\end{array}\right.
$$


Then

$$
\|u\|_{\overline{\mathbf{B}}^{0}(T)}^{2} \lesssim\|\phi\|_{\overline{\mathbf{E}}^{0}}^{2}+\|v\|_{\mathbf{F}^{1}(T)} \cdot\|u\|_{\overline{\mathbf{F}}^{0}(T)}^{2}
$$

Finally, to estimate differences of solutions in $\mathbf{F}^{1}$ we need to differentiate the difference equation. To get bounds for this equation we need a stronger version of Proposition 3.5.

Proposition 3.6. Assume $T \in(0,1], u \in \overline{\mathbf{F}}^{0}(T), u=P_{\geq-10}(u), v \in \mathbf{F}^{1}(T)$, $w_{1}, w_{2}, w_{3} \in \overline{\mathbf{F}}^{0}(T) \cap \mathbf{F}^{1}(T), w_{1}^{\prime}, w_{2}^{\prime}, w_{3}^{\prime} \in \overline{\mathbf{F}}^{0}(T), h \in \overline{\mathbf{F}}^{0}, h=P_{\leq-5}(h)$, and

$$
\left\{\begin{array}{l}
\partial_{t} u+\partial_{x}^{3} u-\partial_{x}^{-1} \partial_{y}^{2} u=P_{\geq-10}\left(v \cdot \partial_{x} u\right)+\sum_{m=1}^{3} P_{\geq-10}\left(w_{m} \cdot w_{m}^{\prime}\right)+P_{\geq-10}(h) ; \\
u(0)=\phi
\end{array}\right.
$$

on $\mathbb{R}^{2} \times(-T, T)$. Then

$$
\|u\|_{\overline{\mathbf{B}}^{0}(T)}^{2} \lesssim\|\phi\|_{\overline{\mathbf{E}}^{0}}^{2}+\|v\|_{\mathbf{F}^{1}(T)} \cdot\|u\|_{\overline{\mathbf{F}}^{0}(T)}^{2}+\sum_{m=1}^{3}\|u\|_{\overline{\mathbf{F}}^{0}(T)}\left\|w_{m}\right\|_{\overline{\mathbf{F}}^{0}(T)}\left\|w_{m}^{\prime}\right\|_{\overline{\mathbf{F}}^{0}(T)} .
$$

We observe that Proposition 3.5 follows from Proposition 3.6 . let $u^{\prime}=P_{\geq-10} u$ and observe that, using (3.15) and the definitions

$$
\left\{\begin{array}{l}
\|u\|_{\overline{\mathbf{B}}^{0}(T)}^{2} \lesssim\left\|u^{\prime}\right\|_{\overline{\mathbf{B}}^{0}(T)}^{2}+C\|\phi\|_{\overline{\mathbf{E}}^{0}}^{2} ; \\
\partial_{x}(u v)=v \cdot \partial_{x} u^{\prime}+P_{\geq-10} v \cdot \partial_{x} P_{\leq-11} u+u \cdot \partial_{x} v+P_{\leq-11} v \cdot \partial_{x} P_{\leq-11} u .
\end{array}\right.
$$

We prove Proposition 3.4 and Proposition 3.6 in section 6 .

\section{Proof of the Main theorem}

In this section we use the linear, bilinear and energy estimates in the previous section to prove Theorem 1.1. Our starting point is a well-posedness result for more regular solutions:

Proposition 4.1. Assume $\phi \in \mathbf{E}^{\infty}$. Then there is $T=T\left(\|\phi\|_{\mathbf{E}^{3}}\right) \in(0,1]$ and a unique solution $u=S_{T}^{\infty}(\phi) \in C\left([-T, T]: \mathbf{E}^{\infty}\right)$ of the initial value problem

$$
\left\{\begin{array}{l}
\partial_{t} u+\partial_{x}^{3} u-\partial_{x}^{-1} \partial_{y}^{2} u+\partial_{x}\left(u^{2} / 2\right)=0 \text { on } \mathbb{R}^{2} \times(-T, T) \\
u(0)=\phi
\end{array}\right.
$$

In addition, for any $\sigma \geq 3$

$$
\sup _{t \in[-T, T]}\|u(t)\|_{\mathbf{E}^{\sigma}} \leq C\left(\sigma,\|\phi\|_{\mathbf{E}^{\sigma}}, \sup _{t \in[-T, T]}\|u(t)\|_{\mathbf{E}^{3}}\right) .
$$


Proposition 4.1 follows by standard energy estimates (see [8]), since

$$
\|\phi\|_{L^{\infty}}+\left\|\partial_{x} \phi\right\|_{L^{\infty}} \lesssim\|\phi\|_{\mathbf{E}^{3}} .
$$

To prove Theorem 1.1 (a), by scaling we may assume that

$$
\|\phi\|_{\mathbf{E}^{1}} \leq \varepsilon_{0} \ll 1 \text {. }
$$

The uniqueness part of Theorem 1.1 (a) follows from Proposition 4.1. For global existence, in view of the conservation laws (1.4), we only need to construct the solution on the time interval $[-1,1]$. In view of Proposition 4.1, it suffices to prove that if $T \in(0,1]$ and $u \in C\left([-T, T]: \mathbf{E}^{\infty}\right)$ is a solution of (4.1) with $\|\phi\|_{\mathbf{E}^{1}} \leq \varepsilon_{0} \ll 1$ then

$$
\sup _{t \in[-T, T]}\|u(t)\|_{\mathbf{E}^{3}} \lesssim\|\phi\|_{\mathbf{E}^{3}} .
$$

We first use a continuity argument to establish an $\mathbf{F}^{1}$ bound on $u$ in the interval $[-T, T]$. By taking $\sigma=1$, it follows from Proposition 3.2 (a), Proposition 3.3 (a), and Proposition 3.4 that for any $T^{\prime} \in[0, T]$ we have

$$
\left\{\begin{array}{l}
\|u\|_{\mathbf{F}^{1}\left(T^{\prime}\right)} \lesssim\|u\|_{\mathbf{B}^{1}\left(T^{\prime}\right)}+\left\|\partial_{x}\left(u^{2}\right)\right\|_{\mathbf{N}^{1}\left(T^{\prime}\right)} \\
\left\|\partial_{x}\left(u^{2}\right)\right\|_{\mathbf{N}^{1}\left(T^{\prime}\right)} \lesssim\|u\|_{\mathbf{F}^{1}\left(T^{\prime}\right)}^{2} \\
\|u\|_{\mathbf{B}^{1}\left(T^{\prime}\right)}^{2} \lesssim\|\phi\|_{\mathbf{E}^{1}}^{2}+\|u\|_{\mathbf{F}^{1}\left(T^{\prime}\right)}^{3}
\end{array}\right.
$$

We denote $X\left(T^{\prime}\right)=\|u\|_{\mathbf{B}^{1}\left(T^{\prime}\right)}+\left\|\partial_{x}\left(u^{2}\right)\right\|_{\mathbf{N}^{1}\left(T^{\prime}\right)}$ and eliminate $\|u\|_{\mathbf{F}^{1}\left(T^{\prime}\right)}$ to obtain

$$
X\left(T^{\prime}\right)^{2} \lesssim\|\phi\|_{\mathbf{E}^{1}}^{2}+X\left(T^{\prime}\right)^{3}+X\left(T^{\prime}\right)^{4}
$$

Assuming that $X\left(T^{\prime}\right)$ is continuous and satisfies

$$
\lim _{T^{\prime} \rightarrow 0} X\left(T^{\prime}\right) \lesssim\|\phi\|_{\mathbf{E}^{1}}
$$

if $\varepsilon_{0}$ is sufficiently small, we would conclude that $X\left(T^{\prime}\right) \lesssim\|\phi\|_{\mathbf{E}^{1}}$. Using (4.4),

$$
\|u\|_{\mathbf{F}^{1}(T)} \lesssim\|\phi\|_{\mathbf{E}^{1}}
$$

To obtain (4.6) and the continuity of $X\left(T^{\prime}\right)$ we first observe that for $u \in$ $C\left([-T, T]: \mathbf{E}^{\infty}\right)$ the mapping $T^{\prime} \rightarrow\|u\|_{\mathbf{B}^{1}\left(T^{\prime}\right)}$ is increasing and continuous on the interval $[-T, T]$ and

$$
\lim _{T^{\prime} \rightarrow 0}\|u\|_{\mathbf{B}^{1}\left(T^{\prime}\right)} \lesssim\|\phi\|_{\mathbf{E}^{1}}
$$

The similar properties of $\left\|\partial_{x}\left(u^{2}\right)\right\|_{\mathbf{N}^{1}\left(T^{\prime}\right)}$ are obtained by applying the following lemma to $v=\partial_{x}\left(u^{2}\right)$.

Lemma 4.2. Assume $T \in(0,1]$ and $v \in C\left([-T, T]: \mathbf{E}^{\infty}\right)$. Then the mapping $T^{\prime} \rightarrow\|v\|_{\mathrm{N}^{1}\left(T^{\prime}\right)}$ is increasing and continuous on the interval $[0, T]$ and

$$
\lim _{T^{\prime} \rightarrow 0}\|v\|_{\mathbf{N}^{1}\left(T^{\prime}\right)}=0 .
$$


Proof of Lemma 4.2. In view of the definitions and (2.21),

$$
\|v\|_{\mathbf{N}^{1}\left(T^{\prime}\right)} \lesssim\left\|p(\xi, \mu) \cdot \mathcal{F}\left(v \cdot \mathbf{1}_{\left[-T^{\prime}, T^{\prime}\right]}(t)\right)\right\|_{L^{2}} \lesssim T^{\prime 1 / 2} \sup _{t \in\left[-T^{\prime}, T^{\prime}\right]}\|v(t)\|_{\mathbf{E}^{1}} .
$$

The limit in (4.8) follows. It remains to prove the continuity of the mapping $T^{\prime} \rightarrow\|v\|_{\mathbf{N}^{1}\left(T^{\prime}\right)}$ at some point $T_{0}^{\prime} \in(0, T]$. We fix $\varepsilon>0$. Let $D_{r}(v)(x, y, t)=$ $v(x, y, t / r), r \in[1 / 2,2]$. Using (4.9) we have

$$
\|v\|_{\mathbf{N}^{1}\left(T^{\prime}\right)}-\left\|D_{T^{\prime} / T_{0}^{\prime}}(v)\right\|_{\mathbf{N}^{1}\left(T^{\prime}\right)} \lesssim \sup _{t \in\left[-T^{\prime}, T^{\prime}\right]}\left\|v(t)-D_{T^{\prime} / T_{0}^{\prime}}(v)(t)\right\|_{\mathbf{E}^{1}} \leq \varepsilon
$$

for any $T^{\prime} \in(0, T]$ sufficiently close to $T_{0}^{\prime}$. Also, using the definitions

$$
\lim _{r \rightarrow 1}\left\|D_{r}(v)\right\|_{\mathbf{N}^{1}\left(r T_{0}^{\prime}\right)}=\|v\|_{\mathbf{N}^{1}\left(T_{0}^{\prime}\right)}
$$

which completes the proof of the lemma.

To prove (4.3) we combine again Proposition 3.2 (a), Proposition 3.3 (a), and Proposition 3.4 with $\sigma=2,3$ to conclude that

$$
\left\{\begin{array}{c}
\|u\|_{\mathbf{F}^{\sigma}(T)} \lesssim\|u\|_{\mathbf{B}^{\sigma}(T)}+\left\|\partial_{x}\left(u^{2}\right)\right\|_{\mathbf{N}^{\sigma}(T)} \\
\left\|\partial_{x}\left(u^{2}\right)\right\|_{\mathbf{N}^{\sigma}(T)} \lesssim\|u\|_{\mathbf{F}^{1}(T)} \cdot\|u\|_{\mathbf{F}^{\sigma}(T)} \\
\|u\|_{\mathbf{B}^{\sigma}(T)}^{2} \lesssim\|\phi\|_{\mathbf{E}^{\sigma}}^{2}+\|u\|_{\mathbf{F}^{1}(T)} \cdot\|u\|_{\mathbf{F}^{\sigma}}^{2}
\end{array}\right.
$$

Using (4.2) and (4.7), it follows that

$$
\|u\|_{\mathbf{F}^{\sigma}(T)} \lesssim\|\phi\|_{\mathbf{E}^{\sigma}} \text { for } \sigma \in\{2,3\} .
$$

Then the inequality (4.3) follows from Lemma 3.1.

We prove now Theorem 1.1 (b). Assume $\phi \in \mathbf{E}^{1}$ is fixed,

$$
\left\{\phi_{n}: n \in \mathbb{Z}_{+}\right\} \subseteq \mathbf{E}^{\infty} \text { and } \lim _{n \rightarrow \infty} \phi_{n}=\phi \text { in } \mathbf{E}^{1} .
$$

It suffices to prove that the sequence $S_{T}^{\infty}\left(\phi_{n}\right) \in C\left([-T, T]: \mathbf{E}^{\infty}\right)$ is a Cauchy sequence in $C\left([-T, T]: \mathbf{E}^{1}\right)$. By scaling, we may assume

$$
\|\phi\|_{\mathbf{E}^{1}} \leq \varepsilon_{0} \ll 1 \text { and }\left\|\phi_{n}\right\|_{\mathbf{E}^{1}} \leq \varepsilon_{0} \ll 1 \text { for any } n \in \mathbb{Z}_{+} .
$$

Using the conservation laws (1.4) it suffices to prove that for any $\delta>0$ there is $M_{\delta}$ such that

$$
\sup _{t \in[-1,1]}\left\|S^{\infty}\left(\phi_{m}\right)(t)-S^{\infty}\left(\phi_{n}\right)(t)\right\|_{\mathbf{E}^{1}} \leq \delta \text { for any } m, n \geq M_{\delta}
$$

For $K \in \mathbb{Z}_{+}$let

$$
\phi_{n}^{K}=P_{\leq K} \phi_{n} .
$$

We show first that for any $K \in \mathbb{Z}_{+}$there is $M_{\delta, K}$ such that

$$
\sup _{t \in[-1,1]}\left\|S^{\infty}\left(\phi_{m}^{K}\right)(t)-S^{\infty}\left(\phi_{n}^{K}\right)(t)\right\|_{\mathbf{E}^{1}} \leq \delta \text { for any } m, n \geq M_{\delta, K} .
$$


Using Theorem 1.1 (a)

$$
\sup _{t \in[-1,1]}\left\|S^{\infty}\left(\phi_{n}^{K}\right)\right\|_{\mathbf{E}^{10}} \leq C(K) \text { for any } n \in \mathbb{Z}_{+} .
$$

Standard energy estimates for the difference equation show that

$$
\sup _{t \in[-1,1]}\left\|S^{\infty}\left(\phi_{m}^{K}\right)(t)-S^{\infty}\left(\phi_{n}^{K}\right)(t)\right\|_{\mathbf{E}^{1}} \leq C^{\prime}(K) \cdot\left\|\phi_{m}-\phi_{n}\right\|_{\mathbf{E}^{1}},
$$

and (4.14) follows.

We show now that for any $\delta>0$ there are $K \in \mathbb{Z}_{+}$and $M_{\delta}$ such that

$$
\sup _{t \in[-1,1]}\left\|S^{\infty}\left(\phi_{n}\right)(t)-S^{\infty}\left(\phi_{n}^{K}\right)(t)\right\|_{\mathbf{E}^{1}} \leq \delta \text { for any } n \geq M_{\delta} .
$$

The main bound (4.13) would follow from (4.14) and (4.15). To prove (4.15) we need to estimate differences of solutions. We summarize our main result in Proposition 4.3 below. The bound (4.15) follows from (4.17) and Lemma 3.1.

$$
\begin{aligned}
\sup _{t \in[-1,1]}\left\|S^{\infty}\left(\phi_{n}\right)(t)-S^{\infty}\left(\phi_{n}^{K}\right)(t)\right\|_{\mathbf{E}^{1}} & \lesssim\left\|S^{\infty}\left(\phi_{n}\right)(t)-S^{\infty}\left(\phi_{n}^{K}\right)(t)\right\|_{\mathbf{F}^{1}(1)} \\
& \lesssim\left\|\phi_{n}-\phi_{n}^{K}\right\|_{\mathbf{E}^{1}}+C\left\|\phi_{n}^{K}\right\|_{\mathbf{E}^{2}}\left\|\phi_{n}-\phi_{n}^{K}\right\|_{\overline{\mathbf{E}}^{0}} \\
& \lesssim\left\|\phi-\phi_{n}\right\|_{\mathbf{E}^{1}}+\left\|\phi-P_{\leq K} \phi\right\|_{\mathbf{E}^{1}} .
\end{aligned}
$$

Proposition 4.3. Let $u_{1}, u_{2} \in \mathbf{F}^{1}(1)$ be solutions to (1.1) with initial data $\phi_{1}, \phi_{2} \in \mathbf{E}^{\infty}$ satisfying

$$
\left\|\phi_{1}\right\|_{\mathbf{E}^{1}}+\left\|\phi_{2}\right\|_{\mathbf{E}^{1}} \leq \varepsilon_{0} \ll 1, \quad \phi_{1}-\phi_{2} \in \overline{\mathbf{E}}^{0} .
$$

Then

$$
\left\|u_{1}-u_{2}\right\|_{\overline{\mathbf{F}}^{0}(1)} \lesssim\left\|\phi_{1}-\phi_{2}\right\|_{\overline{\mathbf{E}}^{0}}
$$

and

$$
\left\|u_{1}-u_{2}\right\|_{\mathbf{F}^{1}(1)} \lesssim\left\|\phi_{1}-\phi_{2}\right\|_{\mathbf{E}^{1}}+\left\|\phi_{1}\right\|_{\mathbf{E}^{2}}\left\|\phi_{1}-\phi_{2}\right\|_{\mathbf{E}^{0}}
$$

Proof of Proposition 4.3. The difference $v=u_{2}-u_{1}$ solves the equation

$$
\left\{\begin{array}{l}
\partial_{t} v+\partial_{x}^{3} v-\partial_{x}^{-1} \partial_{y}^{2} v=-\partial_{x}\left[v\left(u_{1}+u_{2}\right) / 2\right] \\
v(0)=\phi=\phi_{2}-\phi_{1}
\end{array}\right.
$$

By (4.7) we can bound

$$
\left\|u_{1}\right\|_{\mathbf{F}^{1}(1)}+\left\|u_{2}\right\|_{\mathbf{F}^{1}(1)} \lesssim \varepsilon_{0} .
$$

By Proposition 3.2 (b)3, Proposition 3.3 (b), and Proposition 3.5 we obtain

$$
\left\{\begin{array}{l}
\|v\|_{\overline{\mathbf{F}}^{0}(1)} \lesssim\|v\|_{\overline{\mathbf{B}}^{0}(1)}+\left\|\partial_{x}\left[v\left(u_{1}+u_{2}\right) / 2\right]\right\|_{\overline{\mathbf{N}}^{0}(1)} \\
\left\|\partial_{x}\left[v\left(u_{1}+u_{2}\right) / 2\right]\right\|_{\overline{\mathbf{N}}^{0}(1)} \lesssim\|v\|_{\overline{\mathbf{F}}^{0}(1)}\left(\left\|u_{1}\right\|_{\mathbf{F}^{1}(1)}+\left\|u_{2}\right\|_{\mathbf{F}^{1}(1)}\right) \\
\|v\|_{\overline{\mathbf{B}}^{0}(1)}^{2} \lesssim\|\phi\|_{\overline{\mathbf{E}}^{0}}^{2}+\|v\|_{\overline{\mathbf{F}}^{0}(1)}^{2}\left(\left\|u_{1}\right\|_{\mathbf{F}^{1}(1)}+\left\|u_{2}\right\|_{\mathbf{F}^{1}(1)}\right)
\end{array}\right.
$$

\footnotetext{
${ }^{3}$ Clearly, $\partial_{x}\left[v\left(u_{1}+u_{2}\right) / 2\right] \in \overline{\mathbf{N}}^{0}(1)$ since $\partial_{x}\left[v\left(u_{1}+u_{2}\right) / 2\right] \in C\left([-1,1]: \overline{\mathbf{E}}^{0}\right)$.
} 
Combining this with (4.19) we obtain the estimate (4.16).

To prove (4.17) we first use Proposition 3.2 (a) and Proposition 3.3 (a) to obtain

$$
\left\{\begin{array}{l}
\|v\|_{\mathbf{F}^{1}(1)} \lesssim\|v\|_{\mathbf{B}^{1}(1)}+\left\|\partial_{x}\left[v\left(u_{1}+u_{2}\right) / 2\right]\right\|_{\mathbf{N}^{1}(1)} \\
\left\|\partial_{x}\left[v\left(u_{1}+u_{2}\right) / 2\right]\right\|_{\mathbf{N}^{1}(1)} \lesssim\|v\|_{\mathbf{F}^{1}(1)} \cdot\left(\left\|u_{1}\right\|_{\mathbf{F}^{1}(1)}+\left\|u_{2}\right\|_{\mathbf{F}^{1}(1)}\right)
\end{array}\right.
$$

Since $\left\|P_{\leq 0}(v)\right\|_{\mathbf{B}^{1}(1)}=\left\|P_{\leq 0}(\phi)\right\|_{\mathbf{E}^{1}}$, it follows from (4.19) that

$$
\|v\|_{\mathbf{F}^{1}(1)} \lesssim\left\|P_{\geq 1}(v)\right\|_{\mathbf{B}^{1}(1)}+\|\phi\|_{\mathbf{E}^{1}}
$$

By (4.16) and (4.11), for (4.17) it remains to prove the estimate

$$
\left\|P_{\geq 1}(v)\right\|_{\mathbf{B}^{1}(1)}^{2} \lesssim\|\phi\|_{\mathbf{E}^{1}}^{2}+\left\|u_{1}\right\|_{\mathbf{F}^{2}(1)} \cdot\|v\|_{\overline{\mathbf{F}}^{0}(1)}\left(\left\|P_{\geq 1}(v)\right\|_{\mathbf{B}^{1}(1)}+\|\phi\|_{\mathbf{E}^{1}}\right) .
$$

In view of the definitions,

$$
\left\|P_{\geq 1}(v)\right\|_{\mathbf{B}^{1}(1)} \approx\left\|P_{\geq 1}\left(\partial_{x} v\right)\right\|_{\overline{\mathbf{B}}^{0}(1)}+\left\|P_{\geq 1}\left(\partial_{x}^{-1} \partial_{y} v\right)\right\|_{\overline{\mathbf{B}}^{0}(1)} .
$$

Using (4.18) we write the equation for $U_{1}=P_{\geq-10}\left(\partial_{x} v\right)$ in the form

$$
\left\{\begin{array}{l}
\partial_{t} U_{1}+\partial_{x}^{3} U_{1}-\partial_{x}^{-1} \partial_{y}^{2} U_{1}=P_{\geq-10}\left(-u_{2} \cdot \partial_{x} U_{1}\right)+P_{\geq-10}\left(G_{1}\right) \\
U_{1}(0)=P_{\geq-10}\left(\partial_{x} \phi\right)
\end{array}\right.
$$

where

$$
\begin{aligned}
G_{1}= & -P_{\geq-10}\left(u_{2}\right) \cdot \partial_{x}^{2} P_{\leq-11}(v)-P_{\leq-11}\left(u_{2}\right) \cdot \partial_{x}^{2} P_{\leq-11}(v) \\
& -\partial_{x} v \cdot \partial_{x}\left(u_{1}+u_{2}\right)-v \cdot \partial_{x}^{2} u_{1} .
\end{aligned}
$$

It follows from Proposition 3.6 (with $h=-P_{\leq-11}\left(u_{2}\right) \cdot \partial_{x}^{2} P_{\leq-11}(v)$ ) that

$$
\begin{aligned}
\left\|P_{\geq-10}\left(\partial_{x} v\right)\right\|_{\overline{\mathbf{B}}^{0}(1)}^{2} \lesssim\left\|\partial_{x} \phi\right\|_{\overline{\mathbf{E}}^{0}}^{2}+\left\|u_{2}\right\|_{\mathbf{F}^{1}(1)}\left\|P_{\geq-10}\left(\partial_{x} v\right)\right\|_{\overline{\mathbf{F}}^{0}(1)}^{2} \\
+\left\|P_{\geq-10}\left(\partial_{x} v\right)\right\|_{\overline{\mathbf{F}}^{0}(1)} \cdot\left\|\partial_{x} v\right\|_{\overline{\mathbf{F}}^{0}(1)} \cdot\left[\left\|\partial_{x} u_{1}\right\|_{\overline{\mathbf{F}}^{0}(1)}+\left\|\partial_{x} u_{2}\right\|_{\overline{\mathbf{F}}^{0}(1)}\right] \\
+\left\|P_{\geq-10}\left(\partial_{x} v\right)\right\|_{\overline{\mathbf{F}}^{0}(1)} \cdot\|v\|_{\overline{\mathbf{F}}^{0}(1)} \cdot\left\|\partial_{x}^{2} u_{1}\right\|_{\overline{\mathbf{F}}^{0}(1)} \cdot
\end{aligned}
$$

Using (4.19) and (4.20), it follows that

$$
\begin{aligned}
\left\|P_{\geq-10}\left(\partial_{x} v\right)\right\|_{\overline{\mathbf{B}}^{0}(1)}^{2} \lesssim\|\phi\|_{\mathbf{E}^{1}}^{2}+\varepsilon_{0}\left(\left\|P_{\geq 1}(v)\right\|_{\mathbf{B}^{1}(1)}^{2}+\|\phi\|_{\mathbf{E}^{1}}^{2}\right) \\
+\left(\left\|P_{\geq 1}(v)\right\|_{\mathbf{B}^{1}(1)}+\|\phi\|_{\mathbf{E}^{1}}\right) \cdot\|v\|_{\overline{\mathbf{F}}^{0}(1)} \cdot\left\|u_{1}\right\|_{\mathbf{F}^{2}(1)} .
\end{aligned}
$$

Using (4.18) we write the equation for $U_{2}=P_{\geq-10}\left(\partial_{x}^{-1} \partial_{y} v\right)$ in the form

$$
\left\{\begin{array}{l}
\partial_{t} U_{2}+\partial_{x}^{3} U_{2}-\partial_{x}^{-1} \partial_{y}^{2} U_{2}=P_{\geq-10}\left(-u_{2} \cdot \partial_{x} U_{2}\right)+P_{\geq-10} G_{2} \\
U_{2}(0)=P_{\geq-10}\left(\partial_{x}^{-1} \partial_{y} \phi\right)
\end{array}\right.
$$

where

$$
G_{2}=-P_{\geq-10}\left(u_{2}\right) \cdot \partial_{x} P_{\leq-11}\left(\partial_{x}^{-1} \partial_{y} v\right)-P_{\leq-11}\left(u_{2}\right) \cdot \partial_{x} P_{\leq-11}\left(\partial_{x}^{-1} \partial_{y} v\right)-v \cdot \partial_{y} u_{1}
$$


It follows from Proposition 3.6 (with $h=-P_{\leq-11}\left(u_{2}\right) \cdot \partial_{x} P_{\leq-11}\left(\partial_{x}^{-1} \partial_{y} v\right)$ ) that

$$
\begin{aligned}
& \left\|P_{\geq-10}\left(\partial_{x}^{-1} \partial_{y} v\right)\right\|_{\overline{\mathbf{B}}^{0}(1)}^{2} \lesssim\left\|P_{\geq-10}\left(\partial_{x}^{-1} \partial_{y} \phi\right)\right\|_{\overline{\mathbf{E}}^{0}}^{2}+\left\|u_{2}\right\|_{\mathbf{F}^{1}(1)}\|v\|_{\mathbf{F}^{1}(1)}^{2} \\
& +\left\|P_{\geq-10}\left(\partial_{x}^{-1} \partial_{y} v\right)\right\|_{\overline{\mathbf{F}}^{0}(1)} \cdot\|v\|_{\overline{\mathbf{F}}^{0}(1)} \cdot\left\|\partial_{x}\left(\partial_{x}^{-1} \partial_{y} u_{1}\right)\right\|_{\overline{\mathbf{F}}^{0}(1)} .
\end{aligned}
$$

Using (4.19) and (4.20), it follows that

$$
\begin{array}{r}
\left\|P_{\geq-10}\left(\partial_{x}^{-1} \partial_{y} v\right)\right\|_{\overline{\mathbf{B}}^{0}(1)}^{2} \lesssim\|\phi\|_{\mathbf{E}^{1}}^{2}+\varepsilon_{0}\left(\left\|P_{\geq 1}(v)\right\|_{\mathbf{B}^{1}(1)}^{2}+\|\phi\|_{\mathbf{E}^{1}}^{2}\right) \\
+\left(\left\|P_{\geq 1}(v)\right\|_{\mathbf{B}^{1}(1)}+\|\phi\|_{\mathbf{E}^{1}}\right) \cdot\|v\|_{\overline{\mathbf{F}}^{0}(1)} \cdot\left\|u_{1}\right\|_{\mathbf{F}^{2}(1)} .
\end{array}
$$

We add up (4.23) and (4.24) and use (4.22). The bound (4.21) follows.

\section{5. $L^{2}$ BILINEAR ESTIMATES}

For $k \in \mathbb{Z}$ and $l, j \in \mathbb{R}$ let

$$
D_{k, l, j}=\left\{(\xi, \mu, \tau): \xi \in \widetilde{I}_{k},|\mu| \leq 2^{l},|\tau-\omega(\xi, \mu)| \leq 2^{j}\right\},
$$

and let $D_{k, \infty, j}=\cup_{l \in \mathbb{Z}} D_{k, l, j}$.

Lemma 5.1. (a) Assume $k_{1}, k_{2}, k_{3} \in \mathbb{Z}, j_{1}, j_{2}, j_{3} \in \mathbb{Z}_{+}$, and $f_{i}: \mathbb{R}^{3} \rightarrow \mathbb{R}_{+}$are $L^{2}$ functions supported in $D_{k_{i}, \infty, j_{i}}, i=1,2,3$. If

$$
\max \left(j_{1}, j_{2}, j_{3}\right) \leq k_{1}+k_{2}+k_{3}-20
$$

then

$$
\int_{\mathbb{R}^{3}}\left(f_{1} * f_{2}\right) \cdot f_{3} \lesssim 2^{\left(j_{1}+j_{2}+j_{3}\right) / 2} \cdot 2^{-\left(k_{1}+k_{2}+k_{3}\right) / 2} \cdot\left\|f_{1}\right\|_{L^{2}}\left\|f_{2}\right\|_{L^{2}}\left\|f_{3}\right\|_{L^{2}} .
$$

(b)Assume $k_{1}, k_{2}, k_{3} \in \mathbb{Z}, l_{1}, l_{2}, l_{3} \in \mathbb{Z}, j_{1}, j_{2}, j_{3} \in \mathbb{Z}_{+}$, and $f_{i}: \mathbb{R}^{3} \rightarrow \mathbb{R}_{+}$are $L^{2}$ functions supported in $D_{k_{i}, l_{i}, j_{i}}, i=1,2,3$. Then

$$
\int_{\mathbb{R}^{3}}\left(f_{1} * f_{2}\right) \cdot f_{3} \lesssim 2^{\left[\min \left(k_{1}, k_{2}, k_{3}\right)+\min \left(l_{1}, l_{2}, l_{3}\right)+\min \left(j_{1}, j_{2}, j_{3}\right)\right] / 2} \cdot\left\|f_{1}\right\|_{L^{2}}\left\|f_{2}\right\|_{L^{2}}\left\|f_{3}\right\|_{L^{2}} .
$$

Proof of Lemma 5.1. Part (b) follows easily from the Minkowski inequality. Part (a) is proved also in [6]; we reproduce the proof here for the sake of completeness. We observe that

$$
\int_{\mathbb{R}^{3}}\left(f_{1} * f_{2}\right) \cdot f_{3}=\int_{\mathbb{R}^{3}}\left(\tilde{f}_{1} * f_{3}\right) \cdot f_{2}=\int_{\mathbb{R}^{3}}\left(\widetilde{f}_{2} * f_{3}\right) \cdot f_{1},
$$

where $\tilde{f}_{i}(\xi, \mu, \tau)=f_{i}(-\xi,-\mu,-\tau), i=1,2$. In view of the symmetry of (5.2) we may assume

$$
j_{3}=\max \left(j_{1}, j_{2}, j_{3}\right)
$$


We define $f_{i}^{\#}(\xi, \mu, \theta)=f_{i}(\xi, \mu, \theta+\omega(\xi, \mu)), i=1,2,3,\left\|f_{i}^{\#}\right\|_{L^{2}}=\left\|f_{i}\right\|_{L^{2}}$. We rewrite the left-hand side of (5.2) in the form

$$
\begin{aligned}
& \int_{\mathbb{R}^{6}} f_{1}^{\#}\left(\xi_{1}, \mu_{1}, \theta_{1}\right) \cdot f_{2}^{\#}\left(\xi_{2}, \mu_{2}, \theta_{2}\right) \\
& \times f_{3}^{\#}\left(\xi_{1}+\xi_{2}, \mu_{1}+\mu_{2}, \theta_{1}+\theta_{2}+\Omega\left(\left(\xi_{1}, \mu_{1}\right),\left(\xi_{2}, \mu_{2}\right)\right)\right) d \xi_{1} d \xi_{2} d \mu_{1} d \mu_{2} d \theta_{1} d \theta_{2}
\end{aligned}
$$

where

$$
\begin{aligned}
\Omega\left(\left(\xi_{1}, \mu_{1}\right),\left(\xi_{2}, \mu_{2}\right)\right) & =-\omega\left(\xi_{1}+\xi_{2}, \mu_{1}+\mu_{2}\right)+\omega\left(\xi_{1}, \mu_{1}\right)+\omega\left(\xi_{2}, \mu_{2}\right) \\
& =\frac{-\xi_{1} \xi_{2}}{\xi_{1}+\xi_{2}}\left[\left(\sqrt{3} \xi_{1}+\sqrt{3} \xi_{2}\right)^{2}-\left(\frac{\mu_{1}}{\xi_{1}}-\frac{\mu_{2}}{\xi_{2}}\right)^{2}\right] .
\end{aligned}
$$

The functions $f_{i}^{\#}$ are supported in the sets $\left.\{\xi, \mu, \theta): \xi \in \widetilde{I}_{k_{i}}, \mu \in \mathbb{R},|\theta| \leq 2^{j_{i}}\right\}$.

We will prove that if $g_{i}: \mathbb{R}^{2} \rightarrow \mathbb{R}_{+}$are $L^{2}$ functions supported in $\widetilde{\widetilde{I}}_{k_{i}} \times \mathbb{R}$, $i=1,2$, and $g: \mathbb{R}^{3} \rightarrow \mathbb{R}_{+}$is an $L^{2}$ function supported in $\widetilde{I}_{k} \times \mathbb{R} \times\left[-2^{j}, 2^{j}\right]$, $j \leq k_{1}+k_{2}+k-15$, then

$$
\begin{gathered}
\int_{\mathbb{R}^{4}} g_{1}\left(\xi_{1}, \mu_{1}\right) \cdot g_{2}\left(\xi_{2}, \mu_{2}\right) \cdot g\left(\xi_{1}+\xi_{2}, \mu_{1}+\mu_{2}, \Omega\left(\left(\xi_{1}, \mu_{1}\right),\left(\xi_{2}, \mu_{2}\right)\right)\right) d \xi_{1} d \xi_{2} d \mu_{1} d \mu_{2} \\
\lesssim 2^{j / 2} \cdot 2^{-\left(k_{1}+k_{2}+k\right) / 2} \cdot\left\|g_{1}\right\|_{L^{2}}\left\|g_{2}\right\|_{L^{2}}\|g\|_{L^{2}}
\end{gathered}
$$

This suffices for (5.2), in view of (5.5) and (5.6).

To prove (5.8), we observet first that we may assume that the integral in the left-hand side of (5.8) is taken over the set

$$
\mathcal{R}_{++}=\left\{\left(\xi_{1}, \mu_{1}, \xi_{2}, \mu_{2}\right): \xi_{1}+\xi_{2} \geq 0 \text { and } \mu_{1} / \xi_{1}-\mu_{2} / \xi_{2} \geq 0\right\} .
$$

Using the restriction $j \leq k_{1}+k_{2}+k-15$ and (5.7), we may assume also that the integral in the left-hand side of (5.8) is taken over the set

$$
\widetilde{\mathcal{R}}_{++}=\left\{\left(\xi_{1}, \mu_{1}, \xi_{2}, \mu_{2}\right) \in \mathcal{R}_{++}:\left|\sqrt{3}\left(\xi_{1}+\xi_{2}\right)\right|-\left|\mu_{1} / \xi_{1}-\mu_{2} / \xi_{2}\right| \leq 2^{-10}\left|\xi_{1}+\xi_{2}\right|\right\} .
$$

To summarize, it suffices to prove that

$$
\begin{gathered}
\int_{\widetilde{\mathcal{R}}_{++}} g_{1}\left(\xi_{1}, \mu_{1}\right) \cdot g_{2}\left(\xi_{2}, \mu_{2}\right) \cdot g\left(\xi_{1}+\xi_{2}, \mu_{1}+\mu_{2}, \Omega\left(\left(\xi_{1}, \mu_{1}\right),\left(\xi_{2}, \mu_{2}\right)\right)\right) d \xi_{1} d \xi_{2} d \mu_{1} d \mu_{2} \\
\lesssim 2^{j / 2} \cdot 2^{-\left(k_{1}+k_{2}+k\right) / 2} \cdot\left\|g_{1}\right\|_{L^{2}}\left\|g_{2}\right\|_{L^{2}}\|g\|_{L^{2}}
\end{gathered}
$$

We make the changes of variables

$$
\mu_{1}=\sqrt{3} \xi_{1}^{2}+\beta_{1} \xi_{1} \text { and } \mu_{2}=-\sqrt{3} \xi_{2}^{2}+\beta_{2} \xi_{2},
$$

\footnotetext{
${ }^{4}$ There are four identical integrals of this type.
} 
with $d \mu_{1} d \mu_{2}=\xi_{1} \xi_{2} d \beta_{1} d \beta_{2}$. The left-hand side of (5.9) is bounded by

$$
\begin{aligned}
& C 2^{k_{1}+k_{2}} \int_{S} g_{1}\left(\xi_{1}, \sqrt{3} \xi_{1}^{2}+\beta_{1} \xi_{1}\right) \cdot g_{2}\left(\xi_{2},-\sqrt{3} \xi_{2}^{2}+\beta_{2} \xi_{2}\right) \\
& \times g\left(\xi_{1}+\xi_{2}, \sqrt{3} \xi_{1}^{2}-\sqrt{3} \xi_{2}^{2}+\beta_{1} \xi_{1}+\beta_{2} \xi_{2}, \widetilde{\Omega}\left(\left(\xi_{1}, \beta_{1}\right),\left(\xi_{2}, \beta_{2}\right)\right)\right) d \xi_{1} d \xi_{2} d \beta_{1} d \beta_{2},
\end{aligned}
$$

where

$$
S=\left\{\left(\xi_{1}, \beta_{1}, \xi_{2}, \beta_{2}\right): \xi_{1}+\xi_{2} \geq 0 \text { and }\left|\beta_{1}-\beta_{2}\right| \leq 2^{-10}\left(\xi_{1}+\xi_{2}\right)\right\},
$$

and

$$
\widetilde{\Omega}\left(\left(\xi_{1}, \beta_{1}\right),\left(\xi_{2}, \beta_{2}\right)\right)=\xi_{1} \xi_{2}\left(\beta_{1}-\beta_{2}\right)\left(2 \sqrt{3}+\frac{\beta_{1}-\beta_{2}}{\xi_{1}+\xi_{2}}\right) .
$$

We define the functions $h_{i}: \mathbb{R}^{2} \rightarrow \mathbb{R}_{+}$supported in $\widetilde{I}_{k_{i}} \times \mathbb{R}, i=1,2$,

$$
\left\{\begin{array}{l}
h_{1}\left(\xi_{1}, \beta_{1}\right)=2^{k_{1} / 2} \cdot g_{1}\left(\xi_{1}, \sqrt{3} \xi_{1}^{2}+\beta_{1} \xi_{1}\right) ; \\
h_{2}\left(\xi_{2}, \beta_{2}\right)=2^{k_{2} / 2} \cdot g_{2}\left(\xi_{2},-\sqrt{3} \xi_{2}^{2}+\beta_{2} \xi_{2}\right),
\end{array}\right.
$$

with $\left\|h_{i}\right\|_{L^{2}} \approx\left\|g_{i}\right\|_{L^{2}}$. Thus, for (5.8) it suffices to prove that

$$
\begin{aligned}
& 2^{\left(k_{1}+k_{2}\right) / 2} \int_{S} h_{1}\left(\xi_{1}, \beta_{1}\right) \cdot h_{2}\left(\xi_{2}, \beta_{2}\right) \\
& \times g\left(\xi_{1}+\xi_{2}, \sqrt{3} \xi_{1}^{2}-\sqrt{3} \xi_{2}^{2}+\beta_{1} \xi_{1}+\beta_{2} \xi_{2}, \widetilde{\Omega}\left(\left(\xi_{1}, \beta_{1}\right),\left(\xi_{2}, \beta_{2}\right)\right)\right) d \xi_{1} d \xi_{2} d \beta_{1} d \beta_{2} \\
& \lesssim 2^{j / 2} \cdot 2^{-\left(k_{1}+k_{2}+k\right) / 2} \cdot\left\|h_{1}\right\|_{L^{2}}\left\|h_{2}\right\|_{L^{2}}\|g\|_{L^{2}} .
\end{aligned}
$$

To prove (15.13), we may assume without loss of generality that

$$
k_{1} \leq k_{2} .
$$

We make the change of variables $\beta_{1}=\beta_{2}+\beta$. In view of (5.11), (5.12), and the restriction on the support of $g$, we may assume $|\beta| \leq 2^{j-k_{1}-k_{2}+4}$. Thus, the integral in the left-hand side of (5.13) is equal to

$$
\begin{aligned}
& 2^{\left(k_{1}+k_{2}\right) / 2} \int_{\widetilde{S}} h_{1}\left(\xi_{1}, \beta+\beta_{2}\right) \cdot h_{2}\left(\xi_{2}, \beta_{2}\right) \cdot \mathbf{1}_{[-1,1]}\left(\beta / 2^{j-k_{1}-k_{2}+4}\right) \\
& \times g\left(\xi_{1}+\xi_{2}, A\left(\xi_{1}, \xi_{2}, \beta\right)+\beta_{2}\left(\xi_{1}+\xi_{2}\right), B\left(\xi_{1}, \xi_{2}, \beta\right)\right) d \xi_{1} d \xi_{2} d \beta d \beta_{2},
\end{aligned}
$$

where $\widetilde{S}=\left\{\left(\xi_{1}, \xi_{2}, \beta, \beta_{2}\right) \in \mathbb{R}^{4}: \xi_{1}+\xi_{2} \geq 0\right.$ and $\left.|\beta| \leq 2^{-10}\left(\xi_{1}+\xi_{2}\right)\right\}$, and

$$
\left\{\begin{array}{l}
A\left(\xi_{1}, \xi_{2}, \beta\right)=\sqrt{3} \xi_{1}^{2}-\sqrt{3} \xi_{2}^{2}+\beta \xi_{1} \\
B\left(\xi_{1}, \xi_{2}, \beta\right)=\xi_{1} \xi_{2} \beta \cdot\left(2 \sqrt{3}+\beta /\left(\xi_{1}+\xi_{2}\right)\right) .
\end{array}\right.
$$

Let $j^{\prime}=j-k_{1}-k_{2}+4$ and decompose, for $i=1,2$,

$$
h_{i}\left(\xi^{\prime}, \beta^{\prime}\right)=\sum_{m \in \mathbb{Z}} h_{i}\left(\xi^{\prime}, \beta^{\prime}\right) \cdot \mathbf{1}_{[0,1)}\left(\beta^{\prime} / 2^{j^{\prime}}-m\right)=\sum_{m \in \mathbb{Z}} h_{i}^{m}\left(\xi^{\prime}, \beta^{\prime}\right) .
$$


The expression in (5.15) is dominated by

$$
\begin{aligned}
& C 2^{\left(k_{1}+k_{2}\right) / 2} \sum_{\left|m-m^{\prime}\right| \leq 4} \int_{\widetilde{S}} h_{1}^{m}\left(\xi_{1}, \beta+\beta_{2}\right) \cdot h_{2}^{m^{\prime}}\left(\xi_{2}, \beta_{2}\right) \\
& \times g\left(\xi_{1}+\xi_{2}, A\left(\xi_{1}, \xi_{2}, \beta\right)+\beta_{2}\left(\xi_{1}+\xi_{2}\right), B\left(\xi_{1}, \xi_{2}, \beta\right)\right) d \xi_{1} d \xi_{2} d \beta d \beta_{2} .
\end{aligned}
$$

Also, for $i=1,2$,

$$
\left\|h_{i}\right\|_{L^{2}}=\left[\sum_{m \in \mathbb{Z}}\left\|h_{i}^{m}\right\|_{L^{2}}^{2}\right]
$$

Thus, to prove (5.13), we may assume $h_{1}=h_{1}^{m}$ and $h_{2}=h_{2}^{m^{\prime}}$ for some fixed $m, m^{\prime} \in \mathbb{Z}$ with $\left|m-m^{\prime}\right| \leq 4$. To summarize, it suffices to prove that if $F_{i}: \mathbb{R}^{2} \rightarrow$ $[0, \infty)$ are $L^{2}$ functions supported in $\widetilde{I}_{k_{i}} \times \mathbb{R}, g$ is as before, and $m \in \mathbb{Z}$ then

$$
\begin{aligned}
& 2^{\left(k_{1}+k_{2}\right) / 2} \int_{\widetilde{S}} F_{1}\left(\xi_{1}, \beta+\beta_{2}\right) \cdot F_{2}\left(\xi_{2}, \beta_{2}\right) \cdot \mathbf{1}_{[m-1, m+1]}\left(\beta_{2} / 2^{j^{\prime}}\right) \\
& \times g\left(\xi_{1}+\xi_{2}, A\left(\xi_{1}, \xi_{2}, \beta\right)+\beta_{2}\left(\xi_{1}+\xi_{2}\right), B\left(\xi_{1}, \xi_{2}, \beta\right)\right) d \xi_{1} d \xi_{2} d \beta d \beta_{2} \\
& \lesssim 2^{j / 2} \cdot 2^{-\left(k_{1}+k_{2}+k\right) / 2} \cdot\left\|F_{1}\right\|_{L^{2}}\left\|F_{2}\right\|_{L^{2}}\|g\|_{L^{2}} .
\end{aligned}
$$

To prove (5.18) we use the Minkowski inequality in the variables $\left(\xi_{1}, \xi_{2}, \beta\right)$ : with

$$
S^{\prime}=\left\{\left(\xi_{1}, \xi_{2}, \beta\right) \in \mathbb{R}^{3}: \xi_{i} \in \widetilde{I}_{k_{i}}, \xi_{1}+\xi_{2} \geq 0,|\beta| \leq 2^{-10}\left(\xi_{1}+\xi_{2}\right)\right\},
$$

the left-hand side of (15.18) is dominated by

$$
\begin{gathered}
C 2^{\left(k_{1}+k_{2}\right) / 2} \int_{\mathbb{R}} \mathbf{1}_{[m-1, m+1]}\left(\beta_{2} / 2^{j^{\prime}}\right) \cdot\left(\int_{S^{\prime}}\left|F_{1}\left(\xi_{1}, \beta+\beta_{2}\right) \cdot F_{2}\left(\xi_{2}, \beta_{2}\right)\right|^{2} d \xi_{1} d \xi_{2} d \beta\right)^{1 / 2} \\
\quad \times\left(\int_{S^{\prime}}\left|g\left(\xi_{1}+\xi_{2}, A\left(\xi_{1}, \xi_{2}, \beta\right)+\beta_{2}\left(\xi_{1}+\xi_{2}\right), B\left(\xi_{1}, \xi_{2}, \beta\right)\right)\right|^{2} d \xi_{1} d \xi_{2} d \beta\right)^{1 / 2} d \beta_{2} .
\end{gathered}
$$

For (5.18), it is easy to see that it suffices to prove that

$$
\begin{aligned}
\left(\int_{S^{\prime}} \mid g\left(\xi_{1}+\xi_{2}, A\left(\xi_{1}, \xi_{2}, \beta\right)+\beta_{2}\left(\xi_{1}+\xi_{2}\right), B\left(\xi_{1},\right.\right.\right. & \left.\left.\left.\xi_{2}, \beta\right)\right)\left.\right|^{2} d \xi_{1} d \xi_{2} d \beta\right)^{1 / 2} \\
& \lesssim 2^{-\left(k_{1}+k_{2}+k\right) / 2}\|g\|_{L^{2}}
\end{aligned}
$$

for any $\beta_{2} \in \mathbb{R}$. Indeed, assuming (5.20), we can bound the expression in (5.19) by

$$
C 2^{\left(k_{1}+k_{2}\right) / 2} \int_{\mathbb{R}} \mathbf{1}_{[m-1, m+1]}\left(\beta_{2} / 2^{j^{\prime}}\right) \cdot\left\|F_{1}\right\|_{L^{2}}\left\|F_{2}\left(., \beta_{2}\right)\right\|_{L_{\xi_{2}}^{2}} \cdot 2^{-\left(k_{1}+k_{2}+k\right) / 2}\|g\|_{L^{2}} d \beta_{2},
$$

which suffices since $2^{j^{\prime} / 2} 2^{\left(k_{1}+k_{2}\right) / 2} \approx 2^{j / 2}$.

Finally, to prove (5.20), we may assume first that $\beta_{2}=0$. We examine (5.16) and make the change of variable $\beta=\sqrt{3}\left(\xi_{1}+\xi_{2}\right) \cdot \nu$. The left-hand side of (5.20) 
is dominated by

$C\left(2^{k} \int_{S^{\prime \prime}}\left|g\left(\xi_{1}+\xi_{2}, \sqrt{3}\left(\xi_{1}+\xi_{2}\right)\left(\xi_{1}-\xi_{2}+\nu \xi_{1}\right), 3 \xi_{1} \xi_{2}\left(\xi_{1}+\xi_{2}\right) \nu(2+\nu)\right)\right|^{2} d \xi_{1} d \xi_{2} d \nu\right)^{1 / 2}$

where $S^{\prime \prime}=\left\{\left(\xi_{1}, \xi_{2}, \nu\right) \in \mathbb{R}^{3}: \xi_{i} \in \widetilde{I}_{k_{i}},|\nu| \leq 2^{-10}\right\}$. We define the function

$$
h(\xi, x, y)=2^{2 k} \cdot|g(\xi, \sqrt{3} \xi \cdot x, 3 \xi \cdot y)|^{2},
$$

so $\|h\|_{L^{1}} \approx\|g\|_{L^{2}}^{2}$. The expression in (5.21) is dominated by

$$
C 2^{-k / 2}\left(\int_{S^{\prime \prime}}\left|h\left(\xi_{1}+\xi_{2}, \xi_{1}-\xi_{2}+\nu \xi_{1}, \xi_{1} \xi_{2} \cdot \nu(2+\nu)\right)\right| d \xi_{1} d \xi_{2} d \nu\right)^{1 / 2}
$$

Therefore, it remains to prove that

$$
\int_{S^{\prime \prime}}\left|h\left(\xi_{1}+\xi_{2}, \xi_{1}-\xi_{2}+\nu \xi_{1}, \xi_{1} \xi_{2} \cdot \nu(2+\nu)\right)\right| d \xi_{1} d \xi_{2} d \nu \lesssim 2^{-\left(k_{1}+k_{2}\right)}\|h\|_{L^{1}}
$$

for any function $h \in L^{1}\left(\mathbb{R}^{3}\right)$. This is clear since the absolute value of the determinant of the change of variables $\left(\xi_{1}, \xi_{2}, \nu\right) \rightarrow\left[\xi_{1}+\xi_{2}, \xi_{1}-\xi_{2}+\nu \xi_{1}, \xi_{1} \xi_{2} \cdot \nu(2+\nu)\right]$ is equal to $(2+\nu)\left|\xi_{1}\right| \cdot\left|\xi_{2}(2+\nu)+\xi_{1} \nu\right| \approx 2^{k_{1}+k_{2}}$, see (15.14) and the definition of the set $S^{\prime \prime}$.

Lemma 5.2. Assume $k_{1}, k_{2}, k_{3} \in \mathbb{Z}, j_{1}, j_{2}, j_{3} \in \mathbb{Z}_{+}$, and $f_{i}: \mathbb{R}^{3} \rightarrow \mathbb{R}_{+}$are $L^{2}$ functions supported in $D_{k_{i}, \infty, j_{i}}, i=1,2,3$. Then

$$
\int_{\mathbb{R}^{3}}\left(f_{1} * f_{2}\right) \cdot f_{3} \lesssim 2^{\left(j_{1}+j_{2}+j_{3}\right) / 2} \cdot 2^{-\max \left(j_{1}, j_{2}, j_{3}\right) / 2} \cdot\left\|f_{1}\right\|_{L^{2}}\left\|f_{2}\right\|_{L^{2}}\left\|f_{3}\right\|_{L^{2}} .
$$

Proof. Using the symmetry (5.4), we may assume $j_{3}=\max \left(j_{1}, j_{2}, j_{3}\right)$. Then

$$
\int_{\mathbb{R}^{3}}\left(f_{1} * f_{2}\right) \cdot f_{3} \lesssim\left\|f_{3}\right\|_{L^{2}} \cdot\left\|f_{1} * f_{2}\right\|_{L^{2}} \lesssim\left\|f_{3}\right\|_{L^{2}}\left\|\mathcal{F}^{-1}\left(f_{1}\right)\right\|_{L^{4}}\left\|\mathcal{F}^{-1}\left(f_{2}\right)\right\|_{L^{4}}
$$

We use the scale-invariant Strichartz estimate of [1]:

$$
\left\|\int_{\mathbb{R}^{2}} \phi(\xi, \mu) e^{i x \cdot \xi} e^{i y \cdot \mu} e^{i t \cdot \omega(\xi, \mu)} d \xi d \mu\right\|_{L_{x, y, t}^{4}} \lesssim\|\phi\|_{L^{2}}
$$

for any $\phi \in L^{2}\left(\mathbb{R}^{2}\right)$. With $f_{i}^{\#}, i=1,2$, defined as in the proof of Lemma 5.1 , we estimate

$$
\begin{aligned}
& \left\|\int_{\mathbb{R}^{3}} f_{i}(\xi, \mu, \tau) \cdot e^{i x \cdot \xi} e^{i y \cdot \mu} e^{i t \cdot \tau} d \xi d \mu d \tau\right\|_{L_{x, y, t}^{4}} \\
& =\left\|\int_{\mathbb{R}^{3}} f_{i}^{\#}(\xi, \mu, \theta) \cdot e^{i t \cdot \theta} \cdot e^{i x \cdot \xi} e^{i y \cdot \mu} e^{i t \cdot \omega(\xi, \mu)} d \xi d \mu d \theta\right\|_{L_{x, y, t}^{4}} \\
& \lesssim 2^{j_{i} / 2}\left\|f_{i}^{\#}(\xi, \mu, \theta)\right\|_{L^{2}}
\end{aligned}
$$

which gives (5.22). 
As a consequence of Lemma 5.1 and Lemma 5.2, we have the following $L^{2}$ bilinear estimates.

Corollary 5.3. (a) Assume $k_{1}, k_{2}, k \in \mathbb{Z}, j_{1}, j_{2}, j \in \mathbb{Z}_{+}$, and $f_{i}: \mathbb{R}^{3} \rightarrow \mathbb{R}_{+}$are $L^{2}$ functions supported in $D_{k_{i}, \infty, j_{i}}, i=1,2$. Then

$$
\left\|\mathbf{1}_{D_{k, \infty}, j} \cdot\left(f_{1} * f_{2}\right)\right\|_{L^{2}} \lesssim 2^{\left(j_{1}+j_{2}+j\right) / 2}\left(2^{\max \left(j_{1}, j_{2}, j\right)}+2^{k_{1}+k_{2}+k}\right)^{-1 / 2} \cdot\left\|f_{1}\right\|_{L^{2}}\left\|f_{2}\right\|_{L^{2}} .
$$

(b) Assume $k_{1}, k_{2}, k \in \mathbb{Z}, j_{1}, j_{2}, j \in \mathbb{Z}_{+}$, and $f_{i}: \mathbb{R}^{3} \rightarrow \mathbb{R}_{+}$are $L^{2}$ functions supported in $D_{k_{i}, \infty, j_{i}}, i=1,2$. If $k_{1} \leq 100$ then

$$
\begin{aligned}
& \left\|\mathbf{1}_{D_{k, \infty, j}} \cdot\left(f_{1} * f_{2}\right)\right\|_{L^{2}} \lesssim 2^{\left[k_{1}+\min \left(k_{1}, k_{2}, k\right)+\min \left(j_{1}, j_{2}, j\right)\right] / 2} \cdot\left\|p\left(\xi_{1}, \mu_{1}\right) \cdot f_{1}\right\|_{L^{2}}\left\|f_{2}\right\|_{L^{2}} . \\
& \text { If } k_{1} \geq-100 \text { then } \\
& \left\|\mathbf{1}_{D_{k, \infty, j}} \cdot\left(f_{1} * f_{2}\right)\right\|_{L^{2}} \lesssim 2^{\left[2 k_{1}+\min \left(k_{1}, k_{2}, k\right)+\min \left(j_{1}, j_{2}, j\right)\right] / 2} \cdot\left\|p\left(\xi_{1}, \mu_{1}\right) \cdot f_{1}\right\|_{L^{2}}\left\|f_{2}\right\|_{L^{2}} .
\end{aligned}
$$

Proof of Corollary 5.3. Part (a) follows from (5.2) and (5.22). For part (b), recall (see (1.3) ) that $p(\xi, \mu)=1+|\mu| /\left(|\xi|+|\xi|^{2}\right)$. To prove (5.25) we decompose

$$
f_{1}=f_{1, k_{1}}+\sum_{l_{1}=k_{1}+1} f_{1, l_{1}}=f_{1} \cdot \eta_{0}\left(\mu_{1} / 2^{k_{1}}\right)+\sum_{l_{1}=k_{1}+1}^{\infty} f_{1} \cdot \chi_{l_{1}}\left(\mu_{1}\right) .
$$

Using (5.3), the left-hand side of (5.25) is dominated by

$$
\begin{aligned}
& \sum_{l_{1}=k_{1}}^{\infty}\left\|\mathbf{1}_{D_{k, \infty}, j} \cdot\left(f_{1, l_{1}} * f_{2}\right)\right\|_{L^{2}} \lesssim 2^{\left[\min \left(k_{1}, k_{2}, k\right)+\min \left(j_{1}, j_{2}, j\right)\right] / 2}\left\|f_{2}\right\|_{L^{2}} \sum_{l_{1}=k_{1}}^{\infty} 2^{l_{1} / 2}\left\|f_{1, l_{1}}\right\|_{L^{2}} \\
& \lesssim 2^{\left[\min \left(k_{1}, k_{2}, k\right)+\min \left(j_{1}, j_{2}, j\right)\right] / 2}\left\|f_{2}\right\|_{L^{2}} \cdot 2^{k_{1} / 2}\left\|p\left(\xi_{1}, \mu_{1}\right) \cdot f_{1}\right\|_{L^{2}},
\end{aligned}
$$

as desired. To prove (5.26) we decompose

$$
f_{1}=f_{1,2 k_{1}}+\sum_{l_{1}=2 k_{1}+1} f_{1, l_{1}}=f_{1} \cdot \eta_{0}\left(\mu_{1} / 2^{2 k_{1}}\right)+\sum_{l_{1}=2 k_{1}+1}^{\infty} f_{1} \cdot \chi_{l_{1}}\left(\mu_{1}\right) .
$$

Using (5.3), the left-hand side of (5.25) is dominated by

$$
\begin{aligned}
& \sum_{l_{1}=2 k_{1}}^{\infty}\left\|\mathbf{1}_{D_{k, \infty}, j} \cdot\left(f_{1, l_{1}} * f_{2}\right)\right\|_{L^{2}} \lesssim 2^{\left[\min \left(k_{1}, k_{2}, k\right)+\min \left(j_{1}, j_{2}, j\right)\right] / 2}\left\|f_{2}\right\|_{L^{2}} \sum_{l_{1}=2 k_{1}}^{\infty} 2^{l_{1} / 2}\left\|f_{1, l_{1}}\right\|_{L^{2}} \\
& \lesssim 2^{\left[\min \left(k_{1}, k_{2}, k\right)+\min \left(j_{1}, j_{2}, j\right)\right] / 2}\left\|f_{2}\right\|_{L^{2}} \cdot 2^{k_{1}}\left\|p\left(\xi_{1}, \mu_{1}\right) \cdot f_{1}\right\|_{L^{2}},
\end{aligned}
$$

as desired. 


\section{EnERGy estimates}

In this section we prove the energy estimates in Proposition 3.4 and Proposition 3.6. To prove dyadic energy estimates we introduce a new Littlewood-Paley decomposition with smooth symbols. With

$$
\chi_{k}(\xi)=\eta_{0}\left(\xi / 2^{k}\right)-\eta_{0}\left(\xi / 2^{k-1}\right), \quad k \in \mathbb{Z},
$$

let $\widetilde{P}_{k}$ denote the operator on $L^{2}\left(\mathbb{R}^{3}\right)$ defined by the Fourier multiplier $(\xi, \mu, \tau) \rightarrow$ $\chi_{k}(\xi)$. By a slight abuse of notation, we also let $\widetilde{P}_{k}$ denote the operator on $L^{2}\left(\mathbb{R}^{2}\right)$ defined by the Fourier multiplier $(\xi, \mu) \rightarrow \chi_{k}(\xi)$. For $l \in \mathbb{Z}$ let

$$
\widetilde{P}_{\leq l}=\sum_{k \leq l} \widetilde{P}_{k}, \quad \widetilde{P}_{\geq l}=\sum_{k \geq l} \widetilde{P}_{k}
$$

Assume that, for some $k \in \mathbb{Z}$ and $u, v \in C\left([-T, T]: \bar{E}_{k}\right)$

$$
\left\{\begin{array}{l}
\partial_{t} u+\partial_{x}^{3} u-\partial_{x}^{-1} \partial_{y}^{2} u=v \text { on } \mathbb{R}^{2} \times(-T, T) \\
u(0)=\phi
\end{array}\right.
$$

We multiply by $u$ and integrate to conclude that

$$
\sup _{\left|t_{k}\right| \leq T}\left\|u\left(t_{k}\right)\right\|_{L^{2}}^{2} \leq\|\phi\|_{L^{2}}^{2}+\sup _{\left|t_{k}\right| \leq T}\left|\int_{\mathbb{R}^{2} \times\left[0, t_{k}\right]} u \cdot v d x d y d t\right| .
$$

To prove Proposition 3.4 and Proposition 3.6 we need to replace $v$ by the corresponding bilinear expressions. Thus we need to estimate integrals of trilinear forms. However, instead of direct estimates we seek to take advantage of the special form of the nonlinearities. This allows us to place the derivative in the nonlinearity on the lowest frequency factor. We summarize the main dyadic estimates we need in Lemma 6.1 below.

Lemma 6.1. (a) Assume $T \in(0,1], k_{1}, k_{3}, k_{3} \in \mathbb{Z}$ with $\max \left\{k_{1}, k_{2}, k_{3}\right\} \geq 0$, and $u_{i} \in \bar{F}_{k_{i}}(T), i=1,2,3$. Assume in addition that $u_{i} \in F_{k_{i}}(T)$ for some $i \in\{1,2,3\}$. Then

$$
\left|\int_{\mathbb{R}^{2} \times[0, T]} u_{1} u_{2} u_{3} d x d y d t\right| \lesssim 2^{-\min \left(k_{1}, k_{2}, k_{3}\right) / 2} \prod_{i=1}^{3}\left\|u_{k_{i}}\right\|_{\bar{F}_{k_{i}}(T)} .
$$

(b) Assume $T \in(0,1], k \in \mathbb{Z}_{+}, k_{1} \leq k-10, u \in \overline{\mathbf{F}}^{0}(T)$, and $v \in F_{k_{1}}(T)$. Then

$$
\left|\int_{\mathbb{R}^{2} \times[0, T]} \widetilde{P}_{k}(u) \widetilde{P}_{k}\left(\partial_{x} u \cdot \widetilde{P}_{k_{1}}(v)\right) d x d y d t\right| \lesssim 2^{k_{1} / 2}\|v\|_{F_{k_{1}}(T)} \sum_{\left|k^{\prime}-k\right| \leq 10}\left\|\widetilde{P}_{k^{\prime}}(u)\right\|_{F_{k^{\prime}}(T)}{ }
$$

Proof of Lemma 6.1. For part (a), we may assume that $k_{1} \leq k_{2} \leq k_{3}$. In order for the integral to be nontrivial we must also have $\left|k_{2}-k_{3}\right| \leq 4$. The integral in the left-hand side of (6.3) converges absolutely, since one of the factors is in 
$F_{k}(T)$, thus bounded. We fix extensions $\widetilde{u}_{i} \in \bar{F}_{k_{i}}$ such that $\left\|\widetilde{u}_{i}\right\|_{\bar{F}_{k_{i}}} \leq 2\left\|u_{i}\right\|_{\bar{F}_{k_{i}}(T)}$, $i=1,2,3$. Let $\gamma: \mathbb{R} \rightarrow[0,1]$ denote a smooth function supported in $[-1,1]$ with the property that

$$
\sum_{n \in \mathbb{Z}} \gamma^{3}(x-n) \equiv 1, \quad x \in \mathbb{R}
$$

The left-hand side of (6.3) is dominated by

$$
\begin{aligned}
C \sum_{|n| \leq C 2^{k_{3}}} \mid & \int_{\mathbb{R}^{2} \times \mathbb{R}}\left(\gamma\left(2^{k_{3}} t-n\right) \mathbf{1}_{[0, T]}(t) \widetilde{u}_{1}\right) \\
& \times\left(\gamma\left(2^{k_{3}} t-n\right) \mathbf{1}_{[0, T]}(t) \widetilde{u}_{2}\right) \cdot\left(\gamma\left(2^{k_{3}} t-n\right) \mathbf{1}_{[0, T]}(t) \widetilde{u}_{3}\right) d x d y d t \mid .
\end{aligned}
$$

To estimate the integrals in (6.5) we observe that, in view of (15.24), if $k_{1}, k_{2}, k_{3} \in$ $\mathbb{Z}, f_{k_{i}} \in X_{k_{i}}, i=1,2,3$, and $|m| \leq 1$ then

$$
\begin{aligned}
& \mid \int_{\mathbb{R}^{3}} \int_{\mathbb{R}^{3}} m\left(\xi, \xi_{1}\right) \cdot f_{3}(-\xi,-\mu,-\tau) f_{2}\left(\xi-\xi_{1}, \mu-\mu_{1}, \tau-\tau_{1}\right) \\
& \times f_{1}\left(\xi_{1}, \mu_{1}, \tau_{1}\right) d \xi d \mu d \tau d \xi_{1} d \mu_{1} d \tau_{1} \mid \lesssim\left(1+2^{k_{1}+k_{2}+k_{3}}\right)^{-1 / 2} \Pi,
\end{aligned}
$$

where $\Pi=\left\|f_{1}\right\|_{X_{k_{1}}}\left\|f_{2}\right\|_{X_{k_{2}}}\left\|f_{3}\right\|_{X_{k_{3}}}$. In addition, as in (2.5), if $I \subseteq \mathbb{R}$ is an interval, $k \in \mathbb{Z}, f_{k} \in X_{k}$, and $f_{k}^{I}=\mathcal{F}\left(\mathbf{1}_{I}(t) \cdot \mathcal{F}^{-1}\left(f_{k}\right)\right)$ then

$$
\sup _{j \in \mathbb{Z}_{+}} 2^{j / 2}\left\|\eta_{j}(\tau-\omega(\xi, \mu)) \cdot f_{k}^{I}\right\|_{L^{2}} \lesssim\left\|f_{k}\right\|_{X_{k}} .
$$

Thus, using (5.24) again, if $k_{1}, k_{2}, k_{3} \in \mathbb{Z}, f_{k_{i}} \in X_{k_{i}}, i=1,2,3, I_{i} \subseteq \mathbb{R}, i=1,2,3$, are intervals, and $|m| \leq 1$ then

$$
\begin{aligned}
& \mid \int_{\mathbb{R}^{3}} \int_{\mathbb{R}^{3}} m\left(\xi, \xi_{1}\right) \cdot f_{3}^{I_{3}}(-\xi,-\mu,-\tau) f_{2}^{I_{2}}\left(\xi-\xi_{1}, \mu-\mu_{1}, \tau-\tau_{1}\right) \\
& \quad f_{1}^{I_{1}}\left(\xi_{1}, \mu_{1}, \tau_{1}\right) d \xi d \mu d \tau d \xi_{1} d \mu_{1} d \tau_{1} \mid \lesssim\left(1+2^{k_{1}+k_{2}+k_{3}}\right)^{-1 / 2} \max \left(1, k_{1}, k_{2}, k_{3}\right)^{3} \Pi .
\end{aligned}
$$

We apply now the bound (6.7) up to 4 times (for the integers $n$ for which $\left.\gamma\left(2^{k_{3}} t-n\right) \mathbf{1}_{[0, T]}(t) \neq \gamma\left(2^{k_{3}} t-n\right)\right)$ and the bound (6.6) about $2^{k_{3}}$ times to bound the sum in (6.5) by the right-hand side of (6.3) (using also (2.5)). This completes the proof of part (a).

For part (b), we observe first that the expression in the left-hand side of (6.4) is dominated by

$$
\begin{aligned}
& C\left|\int_{\mathbb{R}^{2} \times[0, T]} \widetilde{P}_{k}(u) \cdot \widetilde{P}_{k}\left(\partial_{x} u\right) \cdot \widetilde{P}_{k_{1}}(v) d x d y d t\right| \\
& +C\left|\int_{\mathbb{R}^{2} \times[0, T]} \widetilde{P}_{k}(u) \cdot\left[\widetilde{P}_{k}\left(\partial_{x} u \cdot \widetilde{P}_{k_{1}}(v)\right)-\widetilde{P}_{k}\left(\partial_{x} u\right) \cdot \widetilde{P}_{k_{1}}(v)\right] d x d y d t\right| .
\end{aligned}
$$


We integrate by parts and use (6.3) to conclude that

$$
\left|\int_{\mathbb{R}^{2} \times[0, T]} \widetilde{P}_{k}(u) \cdot \widetilde{P}_{k}\left(\partial_{x} u\right) \cdot \widetilde{P}_{k_{1}}(v) d x d y d t\right| \lesssim 2^{k_{1} / 2}\left\|\widetilde{P}_{k_{1}}(v)\right\|_{\bar{F}_{k_{1}}(T)} \cdot\left\|\widetilde{P}_{k}(u)\right\|_{F_{k}(T)}^{2},
$$

which suffices for (6.4).

To control the term in the second line of (6.8) we fix extensions $\widetilde{u}$ of $u$ and $\widetilde{v}$ of $v$ and use the formula

$$
\begin{aligned}
& \mathcal{F}\left[\widetilde{P}_{k}\left(\widetilde{P}_{k_{1}}(\widetilde{v}) \cdot \partial_{x} \widetilde{u}\right)-\widetilde{P}_{k_{1}}(\widetilde{v}) \cdot \widetilde{P}_{k}\left(\partial_{x} \widetilde{u}\right)\right](\xi, \mu, \tau) \\
& =C \int_{\mathbb{R}^{3}} \mathcal{F}\left(\widetilde{P}_{k_{1}}\left(\partial_{x} \widetilde{v}\right)\right)\left(\xi_{1}, \mu_{1}, \tau_{1}\right) \cdot \mathcal{F}(\widetilde{u})\left(\xi-\xi_{1}, \mu-\mu_{1}, \tau-\tau_{1}\right) \cdot m\left(\xi, \xi_{1}\right) d \xi_{1} d \mu_{1} d \tau_{1},
\end{aligned}
$$

where

$$
\left|m\left(\xi, \xi_{1}\right)\right|=\left|\frac{\left(\xi-\xi_{1}\right)\left(\chi_{k}(\xi)-\chi_{k}\left(\xi-\xi_{1}\right)\right)}{\xi_{1}}\right| \lesssim \sum_{\left|k^{\prime}-k\right| \leq 4} \chi_{k^{\prime}}\left(\xi-\xi_{1}\right) .
$$

The bound (6.4) follows by decomposing the integral in the second line of (6.8) into at most $C 2^{k}$ integrals over time-intervals of length $\approx 2^{-k}$ (as in (6.5)), and using the formula (6.10) and the bounds (6.6) and (6.7) to bound these integrals.

We prove now Proposition 3.4 and Proposition 3.6.

Proof of Proposition 3.4. Recall that $u$ solves the initial-value problem

$$
\left\{\begin{array}{l}
\partial_{t} u+\partial_{x}^{3} u-\partial_{x}^{-1} \partial_{y}^{2} u+\partial_{x}\left(u^{2} / 2\right)=0 \text { on } \mathbb{R}^{2} \times[-T, T] \\
u(0)=\phi
\end{array}\right.
$$

We observe that

$$
\begin{aligned}
& \|u\|_{\mathbf{B}^{\sigma}(T)}^{2}-\left\|P_{\leq 0}(\phi)\right\|_{\mathbf{E}^{\sigma}}^{2} \\
& \lesssim \sum_{k \geq 0} \sup _{t_{k} \in[-T, T]}\left(2^{2 \sigma k}\left\|\widetilde{P}_{k}\left(u\left(t_{k}\right)\right)\right\|_{L^{2}}^{2}+2^{(2 \sigma-2) k}\left\|\widetilde{P}_{k}\left(\partial_{x}^{-1} \partial_{y} u\left(t_{k}\right)\right)\right\|_{L^{2}}^{2}\right)
\end{aligned}
$$

Therefore it suffices to prove that for $\sigma \in\{1,2,3\}$

$$
\begin{aligned}
& \left.\sum_{k \geq 0} \sup _{t_{k} \in[-T, T]} 2^{2 \sigma k}\left\|\widetilde{P}_{k}\left(u\left(t_{k}\right)\right)\right\|_{L^{2}}^{2}+\sum_{k \geq 0} \sup _{t_{k} \in[-T, T]} 2^{(2 \sigma-2) k}\left\|\widetilde{P}_{k}\left(\partial_{x}^{-1} \partial_{y} u\left(t_{k}\right)\right)\right\|_{L^{2}}^{2}\right) \\
& \quad \lesssim\|\phi\|_{\mathbf{E}^{\sigma}}^{2}+\|u\|_{\mathbf{F}^{1}(T)} \cdot\|u\|_{\mathbf{F}^{\sigma}(T)}^{2}
\end{aligned}
$$

We show first that

$$
\sum_{k \geq 0} \sup _{t_{k} \in[-T, T]} 2^{2 \sigma k}\left\|\widetilde{P}_{k}\left(u\left(t_{k}\right)\right)\right\|_{L^{2}}^{2}-2^{2 \sigma k}\left\|\widetilde{P}_{k}(\phi)\right\|_{L^{2}}^{2} \lesssim\|u\|_{\mathbf{F}^{1}(T)} \cdot\|u\|_{\mathbf{F}^{\sigma}(T)}^{2} .
$$


For $k \in \mathbb{Z}_{+}$we use (6.2) and the equation (6.11) to estimate the increment

$$
2^{2 \sigma k}\left\|\widetilde{P}_{k}\left(u\left(t_{k}\right)\right)\right\|_{L^{2}}^{2}-2^{2 \sigma k}\left\|\widetilde{P}_{k}(\phi)\right\|_{L^{2}}^{2} \lesssim 2^{2 \sigma k}\left|\int_{\mathbb{R}^{2} \times\left[0, t_{k}\right]} \widetilde{P}_{k}(u) \widetilde{P}_{k}\left(u \cdot \partial_{x} u\right) d x d y d t\right|
$$

The right-hand side of (6.15) is dominated by

$$
\begin{aligned}
& C 2^{2 \sigma k} \sum_{k_{1} \leq k-10}\left|\int_{\mathbb{R}^{2} \times\left[0, t_{k}\right]} \widetilde{P}_{k}(u) \cdot \widetilde{P}_{k}\left(\widetilde{P}_{k_{1}}(u) \cdot \partial_{x} u\right) d x d y d t\right| \\
& +C 2^{2 \sigma k} \sum_{k_{1} \geq k-9, k_{2} \in \mathbb{Z}}\left|\int_{\mathbb{R}^{2} \times\left[0, t_{k}\right]} \widetilde{P}_{k}^{2}(u) \cdot \widetilde{P}_{k_{1}}(u) \cdot \partial_{x} \widetilde{P}_{k_{2}}(u) d x d y d t\right| .
\end{aligned}
$$

Using (6.4), the sum in the first line of (6.16) is dominated by

$$
C\|u\|_{\mathbf{F}^{1}(T)} \cdot \sum_{\left|k^{\prime}-k\right| \leq 10} 2^{2 \sigma k^{\prime}}\left\|\widetilde{P}_{k^{\prime}}(u)\right\|_{F_{k^{\prime}}(T)}^{2} .
$$

Using (6.3), the sum in the second line of $(\underline{6.16})$ is dominated by

$$
\begin{aligned}
& C 2^{2 \sigma k} \sum_{\left|k_{1}-k\right| \leq 10, k_{2} \leq k+10} 2^{k_{2} / 2}\left\|\widetilde{P}_{k}(u)\right\|_{\bar{F}_{k}(T)}\left\|\widetilde{P}_{k_{1}}(u)\right\|_{\bar{F}_{k_{1}}(T)}\left\|\widetilde{P}_{k_{2}}(u)\right\|_{\bar{F}_{k_{2}}(T)} \\
& +C 2^{2 \sigma k} \sum_{k_{1} \geq k+10,\left|k_{2}-k_{1}\right| \leq 10} 2^{k_{2}-k / 2}\left\|\widetilde{P}_{k}(u)\right\|_{\bar{F}_{k}(T)}\left\|\widetilde{P}_{k_{1}}(u)\right\|_{\bar{F}_{k_{1}}(T)}\left\|\widetilde{P}_{k_{2}}(u)\right\|_{\bar{F}_{k_{2}}(T)} \\
& \lesssim\|u\|_{\mathbf{F}^{1}(T)} \cdot \sum_{\left|k^{\prime}-k\right| \leq 20} 2^{2 \sigma k^{\prime}}\left\|\widetilde{P}_{k^{\prime}}(u)\right\|_{\bar{F}_{k^{\prime}}(T)}^{2}+2^{k / 2}\left\|\widetilde{P}_{k}(u)\right\|_{\bar{F}_{k}(T)} \cdot\|u\|_{\mathbf{F}^{\sigma}(T)}^{2} .
\end{aligned}
$$

The bound (6.14) follows.

We show now that

$$
\begin{array}{r}
\sum_{k \geq 0} \sup _{t_{k} \in[-T, T]} 2^{(2 \sigma-2) k}\left\|\widetilde{P}_{k}\left(\partial_{x}^{-1} \partial_{y} u\left(t_{k}\right)\right)\right\|_{L^{2}}^{2}-2^{(2 \sigma-2) k}\left\|\widetilde{P}_{k}\left(\partial_{x}^{-1} \partial_{y} \phi\right)\right\|_{L^{2}}^{2} \\
\lesssim\|u\|_{\mathbf{F}^{1}(T)} \cdot\|u\|_{\mathbf{F}^{\sigma}(T)}^{2}
\end{array}
$$

For $k \in \mathbb{Z}_{+}$and $t_{k} \in[-T, T]$ we use (6.2) and the the equation (6.11) to estimate the increment

$$
\begin{aligned}
& 2^{(2 \sigma-2) k}\left\|\widetilde{P}_{k}\left(\partial_{x}^{-1} \partial_{y} u\left(t_{k}\right)\right)\right\|_{L^{2}}^{2}-2^{(2 \sigma-2) k}\left\|\widetilde{P}_{k}\left(\partial_{x}^{-1} \partial_{y} \phi\right)\right\|_{L^{2}}^{2} \\
& \quad \lesssim 2^{(2 \sigma-2) k}\left|\int_{\mathbb{R}^{2} \times\left[0, t_{k}\right]} \widetilde{P}_{k}\left(\partial_{x}^{-1} \partial_{y} u\right) \widetilde{P}_{k}\left(u \cdot \partial_{y} u\right) d x d y d t\right| .
\end{aligned}
$$


The right-hand side of $(\underline{6.18})$ is dominated by

$$
\begin{aligned}
& C 2^{(2 \sigma-2) k} \sum_{k_{1} \leq k-10}\left|\int_{\mathbb{R}^{2} \times\left[0, t_{k}\right]} \widetilde{P}_{k}(v) \cdot \widetilde{P}_{k}\left(\widetilde{P}_{k_{1}}(u) \cdot \partial_{x} v\right) d x d y d t\right| \\
& +C 2^{(2 \sigma-2) k} \sum_{k_{1} \geq k-9, k_{2} \in \mathbb{Z}}\left|\int_{\mathbb{R}^{2} \times\left[0, t_{k}\right]} \widetilde{P}_{k}^{2}(v) \cdot \widetilde{P}_{k_{1}}(u) \cdot \partial_{x} \widetilde{P}_{k_{2}}(v) d x d y d t\right|,
\end{aligned}
$$

where $v=\partial_{x}^{-1} \partial_{y} u$. Using (6.4), the sum in the first line of (6.19) is dominated by

$$
C\|u\|_{\mathbf{F}^{1}(T)} \cdot \sum_{\left|k^{\prime}-k\right| \leq 10} 2^{(2 \sigma-2) k^{\prime}}\left\|\widetilde{P}_{k^{\prime}}\left(\partial_{x}^{-1} \partial_{y} u\right)\right\|_{F_{k^{\prime}}(T)}^{2}
$$

Using (6.3), the sum in the second line of (6.19) is dominated by

$$
\begin{aligned}
& C 2^{(2 \sigma-2) k} \sum_{\left|k_{1}-k\right| \leq 10, k_{2} \leq k+10} 2^{k_{2} / 2}\left\|\widetilde{P}_{k}(v)\right\|_{\bar{F}_{k}(T)}\left\|\widetilde{P}_{k_{1}}(u)\right\|_{\bar{F}_{k_{1}}(T)}\left\|\widetilde{P}_{k_{2}}(v)\right\|_{\bar{F}_{k_{2}}(T)} \\
& +C 2^{(2 \sigma-2) k} \sum_{k_{1} \geq k+10,\left|k_{2}-k_{1}\right| \leq 10} 2^{k_{2}-k / 2}\left\|\widetilde{P}_{k}(v)\right\|_{\bar{F}_{k}(T)}\left\|\widetilde{P}_{k_{1}}(u)\right\|_{\bar{F}_{k_{1}}(T)}\left\|\widetilde{P}_{k_{2}}(v)\right\|_{\bar{F}_{k_{2}}(T)} \\
& \lesssim\|u\|_{\mathbf{F}^{1}(T)} \cdot \sum_{\left|k^{\prime}-k\right| \leq 20} 2^{2 \sigma k^{\prime}}\left\|\widetilde{P}_{k^{\prime}}(u)\right\|_{F_{k^{\prime}}(T)}^{2}+C 2^{k / 2}\left\|\widetilde{P}_{k}(u)\right\|_{F_{k}(T)} \cdot\|u\|_{\mathbf{F}^{\sigma}(T)}^{2} .
\end{aligned}
$$

The bound (6.17) follows, which completes the proof of Proposition 3.4.

Proof of Proposition 3.6. Recall that $u=P_{\geq-10}(u)$ solves the equation

$$
\left\{\begin{array}{l}
\partial_{t} u+\partial_{x}^{3} u-\partial_{x}^{-1} \partial_{y}^{2} u=P_{\geq-10}\left(v \cdot \partial_{x} u\right)+\sum_{m=1}^{3} P_{\geq-10}\left(w_{m} \cdot w_{m}^{\prime}\right)+P_{\geq-10}(h) \\
u(0)=\phi
\end{array}\right.
$$

on $\mathbb{R}^{2} \times(-T, T)$. It suffices to prove that

$$
\begin{aligned}
& \sum_{k \geq 0} \sup _{t_{k} \in[-T, T]}\left(\left\|\widetilde{P}_{k}\left(u\left(t_{k}\right)\right)\right\|_{L^{2}}^{2}-\left\|\widetilde{P}_{k}(\phi)\right\|_{L^{2}}^{2}\right) \\
& \lesssim\|v\|_{\mathbf{F}^{1}(T)} \cdot\|u\|_{\overline{\mathbf{F}}^{0}(T)}^{2}+\sum_{m=1}^{3}\|u\|_{\overline{\mathbf{F}}^{0}(T)}\left\|w_{m}\right\|_{\overline{\mathbf{F}}^{0}(T)}\left\|w_{m}^{\prime}\right\|_{\overline{\mathbf{F}}^{0}(T)}
\end{aligned}
$$


Using (6.2) and the equation (6.20), for $k \geq 0$

$$
\begin{aligned}
\left\|\widetilde{P}_{k}\left(u\left(t_{k}\right)\right)\right\|_{L^{2}}^{2}- & \left\|\widetilde{P}_{k}(\phi)\right\|_{L^{2}}^{2} \lesssim\left|\int_{\mathbb{R}^{2} \times\left[0, t_{k}\right]} \widetilde{P}_{k}(u) \cdot \widetilde{P}_{k}\left(\widetilde{P}_{\leq k-10}(v) \cdot \partial_{x} u\right) d x d y d t\right| \\
& +\left|\int_{\mathbb{R}^{2} \times\left[0, t_{k}\right]} \widetilde{P}_{k}^{2}(u) \cdot \partial_{x} u \cdot \widetilde{P}_{\geq k-9}(v) d x d y d t\right| \\
& +\sum_{m=1}^{3}\left|\int_{\mathbb{R}^{2} \times\left[0, t_{k}\right]} \widetilde{P}_{k}^{2}(u) \cdot w_{m} \cdot w_{m}^{\prime} d x d y d t\right|
\end{aligned}
$$

We observe that the term $P_{\geq-10}(h)$ plays no role in the proof of (6.21) (this term is needed, however, to prove the bounds (4.23) and (4.24)).

Using (6.4),

$$
\sum_{k \geq 0}\left|\int_{\mathbb{R}^{2} \times\left[0, t_{k}\right]} \widetilde{P}_{k}(u) \cdot \widetilde{P}_{k}\left(\widetilde{P}_{\leq k-10}(v) \cdot \partial_{x} u\right) d x d y d t\right| \lesssim\|v\|_{\mathbf{F}^{1}(T)}\|u\|_{\overline{\mathbf{F}}^{0}}^{2} .
$$

Using (6.3),

$$
\begin{aligned}
& \sum_{k \geq 0}\left|\int_{\mathbb{R}^{2} \times\left[0, t_{k}\right]} \widetilde{P}_{k}^{2}(u) \cdot \partial_{x} u \cdot \widetilde{P}_{\geq k-9}(v) d x d y d t\right| \\
& \lesssim \sum_{k \geq 0} \sum_{k_{2} \geq k-9} \sum_{k_{1} \leq k_{2}+20}\left|\int_{\mathbb{R}^{2} \times\left[0, t_{k}\right]} \widetilde{P}_{k}^{2}(u) \cdot \partial_{x} \widetilde{P}_{k_{1}}(u) \cdot \widetilde{P}_{k_{2}}(v) d x d y d t\right| \\
& \lesssim \sum_{k \geq 0} \sum_{k_{2} \geq k-9} \sum_{k_{1} \leq k_{2}+20} 2^{k_{1}-\min \left(k_{1}, k\right) / 2} \cdot\left\|\widetilde{P}_{k}(u)\right\|_{\bar{F}_{k}(T)}\left\|\widetilde{P}_{k_{1}}(u)\right\|_{\bar{F}_{k_{1}}(T)}\left\|\widetilde{P}_{k_{2}}(v)\right\|_{\bar{F}_{k_{2}}(T)} \\
& \lesssim\|v\|_{\mathbf{F}^{1}(T)}\|u\|_{\overline{\mathbf{F}}^{0}}^{2} .
\end{aligned}
$$

Using (‥3), with $k_{\text {med }}=k+k_{1}+k_{2}-\min \left(k, k_{1}, k_{2}\right)-\max \left(k, k_{1}, k_{2}\right), k_{\max }=$ $\max \left(k, k_{1}, k_{2}\right)$

$$
\begin{aligned}
& \sum_{k \geq 0}\left|\int_{\mathbb{R}^{2} \times\left[0, t_{k}\right]} \widetilde{P}_{k}^{2}(u) \cdot w_{m} \cdot w_{m}^{\prime} d x d y d t\right| \\
& \lesssim \sum_{k, k_{1}, k_{2} \in \mathbb{Z}}\left|\int_{\mathbb{R}^{2} \times\left[0, t_{k}\right]} \widetilde{P}_{k}^{2}(u) \cdot \widetilde{P}_{k_{1}}\left(w_{m}\right) \cdot \widetilde{P}_{k_{2}}\left(w_{m}^{\prime}\right) d x d y d t\right| \\
& \lesssim \sum_{\left|k_{\operatorname{med}}-k_{\max }\right| \leq 10} 2^{-\min \left(k, k_{1}, k_{2}\right) / 2}\left\|\widetilde{P}_{k}(u)\right\|_{\bar{F}_{k}(T)}\left\|\widetilde{P}_{k_{1}}\left(w_{m}\right)\right\|_{\bar{F}_{k_{1}}(T)}\left\|\widetilde{P}_{k_{2}}\left(w_{m}^{\prime}\right)\right\|_{\bar{F}_{k_{2}}(T)} \\
& \lesssim\|u\|_{\overline{\mathbf{F}}^{0}(T)}\left\|w_{m}\right\|_{\overline{\mathbf{F}}^{0}(T)}\left\|w_{m}^{\prime}\right\|_{\overline{\mathbf{F}}^{0}(T)} .
\end{aligned}
$$

This last inequality uses the fact that, for any $v \in \overline{\mathbf{F}}^{0}(T)$,

$$
\sum_{k \in \mathbb{Z}} 2^{-k / 2}\left\|\widetilde{P}_{k}(v)\right\|_{\bar{F}_{k}(T)} \lesssim\|v\|_{\overline{\mathbf{F}}^{0}(T)},
$$


which is the main reason for the low-frequency condition on functions in $\overline{\mathbf{F}}^{0}$.

The main bound (6.21) follows, which completes the proof of the proposition.

\section{Dyadic Bilinear estimates, I}

In this section we prove several dyadic bounds which are used in the proof of Proposition 3.3 (a). We estimate first Low $\times$ High $\rightarrow$ High interactions.

Lemma 7.1. Assume $k, k_{1}, k_{2} \in \mathbb{Z}, k_{1} \leq k_{2}, k_{1} \leq 0, k \geq 0,\left|k_{2}-k\right| \leq 40$, $u_{k_{1}} \in F_{k_{1}}$ and $v_{k_{2}} \in F_{k_{2}}$. Then

$$
\left\|P_{k}\left(\partial_{x}\left(u_{k_{1}} v_{k_{2}}\right)\right)\right\|_{N_{k}} \lesssim 2^{k_{1}} \cdot\left\|u_{k_{1}}\right\|_{F_{k_{1}}} \cdot\left\|v_{k_{2}}\right\|_{F_{k_{2}}} \cdot
$$

Proof of Lemma 7.1. Using the definitions and (2.21), the left-hand side of (7.1) is dominated by

$$
\begin{aligned}
C \sup _{t_{k} \in \mathbb{R}} \| p(\xi, \mu) & \left(\tau-\omega(\xi, \mu)+i 2^{k}\right)^{-1} \cdot 2^{k} \mathbf{1}_{I_{k}}(\xi) . \\
& \mathcal{F}\left[u_{k_{1}} \cdot \eta_{0}\left(2^{k}\left(t-t_{k}\right)\right)\right] * \mathcal{F}\left[v_{k_{2}} \cdot \eta_{0}\left(2^{k}\left(t-t_{k}\right)\right)\right] \|_{X_{k}} .
\end{aligned}
$$

Let $f_{k_{1}}=\mathcal{F}\left[u_{k_{1}} \cdot \eta_{0}\left(2^{k}\left(t-t_{k}\right)\right)\right]$ and $f_{k_{2}}=\mathcal{F}\left[v_{k_{2}} \cdot \eta_{0}\left(2^{k}\left(t-t_{k}\right)\right)\right]$. Using the bounds (2.4) and (2.21), it suffices to prove that if $j_{1}, j_{2} \geq k$, and $f_{k_{i}, j_{i}}: \mathbb{R}^{3} \rightarrow \mathbb{R}_{+}$are supported in $D_{k_{i}, \infty, j_{i}}, i=1,2$, then

$$
\begin{aligned}
& 2^{k} \sum_{j \geq k} 2^{-j / 2}\left\|\mathbf{1}_{D_{k, \infty}, j} \cdot p(\xi, \mu) \cdot\left(f_{k_{1}, j_{1}} * f_{k_{2}, j_{2}}\right)\right\|_{L^{2}} \\
& \quad \lesssim 2^{k_{1}} \cdot 2^{j_{1} / 2}\left\|p\left(\xi_{1}, \mu_{1}\right) \cdot f_{k_{1}, j_{1}}\right\|_{L^{2}} \cdot 2^{j_{2} / 2}\left\|p\left(\xi_{2}, \mu_{2}\right) \cdot f_{k_{2}, j_{2}}\right\|_{L^{2}} .
\end{aligned}
$$

Since $j, j_{1}, j_{2} \geq k$ it suffices to prove the $L^{2}$ product estimate

$$
\begin{aligned}
& \left\|\mathbf{1}_{D_{k, \infty}, j} \cdot p(\xi, \mu) \cdot\left(f_{k_{1}, j_{1}} * f_{k_{2}, j_{2}}\right)\right\|_{L^{2}} \\
& \lesssim 2^{k_{1}} \cdot 2^{\min \left(j_{1}, j_{2}\right) / 2}\left\|p\left(\xi_{1}, \mu_{1}\right) \cdot f_{k_{1}, j_{1}}\right\|_{L^{2}} \cdot\left\|p\left(\xi_{2}, \mu_{2}\right) \cdot f_{k_{2}, j_{2}}\right\|_{L^{2}} .
\end{aligned}
$$

Using the obvious bound

$$
p(\xi, \mu) \lesssim\left|\xi_{1}\right||\xi|^{-2} p\left(\xi_{1}, \mu_{1}\right)+p\left(\xi_{2}, \mu_{2}\right),
$$

this is a consequence the estimates

$$
\left\|\mathbf{1}_{D_{k, \infty}, j} \cdot\left(f_{k_{1}, j_{1}} * f_{k_{2}, j_{2}}\right)\right\|_{L^{2}} \lesssim 2^{k_{1} / 2+k_{2}} 2^{\min \left(j_{1}, j_{2}\right) / 2}\left\|f_{k_{1}, j_{1}}\right\|_{L^{2}}\left\|p\left(\xi_{2}, \mu_{2}\right) f_{k_{2}, j_{2}}\right\|_{L^{2}},
$$

and

$$
\left\|\mathbf{1}_{D_{k, \infty, j}} \cdot\left(f_{k_{1}, j_{1}} * f_{k_{2}, j_{2}}\right)\right\|_{L^{2}} \lesssim 2^{k_{1}} \cdot 2^{\min \left(j_{1}, j_{2}\right) / 2}\left\|p\left(\xi_{1}, \mu_{1}\right) f_{k_{1}, j_{1}}\right\|_{L^{2}} \cdot\left\|f_{k_{2}, j_{2}}\right\|_{L^{2}}
$$

which follow from (5.26) and (5.25) repectively. 
Lemma 7.2. Assume $k, k_{1}, k_{2} \in \mathbb{Z}, k_{1} \leq k_{2}, k_{1} \geq 0, k \geq 0,\left|k_{2}-k\right| \leq 40$, $u_{k_{1}} \in F_{k_{1}}$, and $v_{k_{2}} \in F_{k_{2}}$. Then

$$
\left\|P_{k}\left(\partial_{x}\left(u_{k_{1}} v_{k_{2}}\right)\right)\right\|_{N_{k}} \lesssim\left(1+k_{1}\right) 2^{-k_{1} / 2} \cdot\left\|u_{k_{1}}\right\|_{F_{k_{1}}} \cdot\left\|v_{k_{2}}\right\|_{F_{k_{2}}} .
$$

Proof of Lemma 7.2. As in the proof of Lemma 7.1, using the definitions and the bounds (2.4) and (2.21), it suffices to prove that if $j_{1}, j_{2} \geq k$ and $f_{k_{i}, j_{i}}: \mathbb{R}^{3} \rightarrow \mathbb{R}_{+}$ are supported in $D_{k_{i}, \infty, j_{i}}, i=1,2$, then

$$
\begin{aligned}
2^{k} & \sum_{j \geq k} 2^{-j / 2}\left\|\mathbf{1}_{D_{k, \infty}, j} \cdot p(\xi, \mu) \cdot\left(f_{k_{1}, j_{1}} * f_{k_{2}, j_{2}}\right)\right\|_{L^{2}} \\
& \lesssim\left(1+k_{1}\right) 2^{-k_{1} / 2} \cdot 2^{j_{1} / 2}\left\|p\left(\xi_{1}, \mu_{1}\right) \cdot f_{k_{1}, j_{1}}\right\|_{L^{2}} \cdot 2^{j_{2} / 2}\left\|p\left(\xi_{2}, \mu_{2}\right) \cdot f_{k_{2}, j_{2}}\right\|_{L^{2}} .
\end{aligned}
$$

Since $j, j_{1}, j_{2} \geq k$, the large modulations $j \geq k+4 k_{1}$ in the output are controlled by the $L^{2}$ product estimate

$$
\begin{aligned}
& \left\|\mathbf{1}_{D_{k, \infty, j}} \cdot p(\xi, \mu) \cdot\left(f_{k_{1}, j_{1}} * f_{k_{2}, j_{2}}\right)\right\|_{L^{2}} \\
& \quad \lesssim 2^{3 k_{1} / 2} \cdot 2^{\min \left(j_{1}, j_{2}\right) / 2}\left\|p\left(\xi_{1}, \mu_{1}\right) \cdot f_{k_{1}, j_{1}}\right\|_{L^{2}} \cdot\left\|p\left(\xi_{2}, \mu_{2}\right) \cdot f_{k_{2}, j_{2}}\right\|_{L^{2}} .
\end{aligned}
$$

In this case we have

$$
p(\xi, \mu) \lesssim\left|\xi_{1}\right|^{2}|\xi|^{-2} p\left(\xi_{1}, \mu_{1}\right)+p\left(\xi_{2}, \mu_{2}\right) .
$$

Hence (7.7) is a consequence of the estimates

$$
\left\|\mathbf{1}_{D_{k, \infty, j}} \cdot\left(f_{k_{1}, j_{1}} * f_{k_{2}, j_{2}}\right)\right\|_{L^{2}} \lesssim 2^{k_{1} / 2+k_{2}} 2^{\min \left(j_{1}, j_{2}\right) / 2}\left\|f_{k_{1}, j_{1}}\right\|_{L^{2}}\left\|p\left(\xi_{2}, \mu_{2}\right) f_{k_{2}, j_{2}}\right\|_{L^{2}}
$$

and

$$
\left\|\mathbf{1}_{D_{k, \infty}, j} \cdot\left(f_{k_{1}, j_{1}} * f_{k_{2}, j_{2}}\right)\right\|_{L^{2}} \lesssim 2^{3 k_{1} / 2} 2^{\min \left(j_{1}, j_{2}\right) / 2}\left\|p\left(\xi_{1}, \mu_{1}\right) f_{k_{1}, j_{1}}\right\|_{L^{2}} \cdot\left\|f_{k_{2}, j_{2}}\right\|_{L^{2}},
$$

which follow from (5.26).

It remains to estimate the small modulations $k \leq j \leq k+4 k_{1}$ in the output. There are about $1+k_{1}$ possible values for $j$, therefore we need to prove that

$$
\begin{aligned}
& \left\|\mathbf{1}_{D_{k, \infty, j}} \cdot p(\xi, \mu) \cdot\left(f_{k_{1}, j_{1}} * f_{k_{2}, j_{2}}\right)\right\|_{L^{2}} \\
& \quad \lesssim 2^{-k_{1} / 2-k} \cdot 2^{\left(j+j_{1}+j_{2}\right) / 2}\left\|p\left(\xi_{1}, \mu_{1}\right) \cdot f_{k_{1}, j_{1}}\right\|_{L^{2}} \cdot\left\|p\left(\xi_{2}, \mu_{2}\right) \cdot f_{k_{2}, j_{2}}\right\|_{L^{2}} .
\end{aligned}
$$

We observe that

$$
\left(\frac{\mu_{2}}{\xi_{2}}-\frac{\mu}{\xi}\right)^{2} \lesssim\left|\xi_{1}\right|^{2}+\left|\xi_{1}\right||\xi|^{-2}\left|\Omega\left(\left(\xi_{1}, \mu_{1}\right),\left(\xi_{2}, \mu_{2}\right)\right)\right|
$$

which leads to

$$
\frac{|\mu|}{|\xi|} \lesssim \frac{\left|\mu_{2}\right|}{\left|\xi_{2}\right|}+\left|\xi_{2}\right|+\left|\xi_{1}\right|^{\frac{1}{2}}|\xi|^{-1}\left|\Omega\left(\left(\xi_{1}, \mu_{1}\right),\left(\xi_{2}, \mu_{2}\right)\right)\right|^{1 / 2}
$$

therefore

$$
p(\xi, \mu) \lesssim p\left(\xi_{2}, \mu_{2}\right)+2^{k_{1} / 2} 2^{-2 k_{2}} 2^{\max \left(j_{1}, j_{2}, j\right) / 2} .
$$


We eliminate the expression $p(\xi, \mu)$ on the left using (7.11), neglecting the remaining $p(\xi, \mu)$ factors on the right. Then it suffices to show that

$$
\left\|\mathbf{1}_{D_{k, \infty, j}} \cdot\left(f_{k_{1}, j_{1}} * f_{k_{2}, j_{2}}\right)\right\|_{L^{2}} \lesssim 2^{-k_{1} / 2-k} 2^{\left(j+j_{1}+j_{2}\right) / 2}\left\|f_{k_{1}, j_{1}}\right\|_{L^{2}}\left\|f_{k_{2}, j_{2}}\right\|_{L^{2}},
$$

and

$$
\left\|\mathbf{1}_{D_{k, \infty, j}} \cdot\left(f_{k_{1}, j_{1}} * f_{k_{2}, j_{2}}\right)\right\|_{L^{2}} \lesssim 2^{-k_{1}+k_{2}} 2^{\left(j+j_{1}+j_{2}\right) / 2} 2^{-\max \left(j_{1}, j_{2}, j\right) / 2}\left\|f_{k_{1}, j_{1}}\right\|_{L^{2}}\left\|f_{k_{2}, j_{2}}\right\|_{L^{2}} .
$$

Both these estimates follow from (5.24).

We estimate now High $\times$ High $\rightarrow$ Low interactions. Let $\gamma: \mathbb{R} \rightarrow[0,1]$ denote a smooth function supported in $[-1,1]$ with the property that $\sum_{m \in \mathbb{Z}} \gamma^{2}(x-m) \equiv 1$, $x \in \mathbb{R}$.

Lemma 7.3. Assume $k, k_{1}, k_{2} \in \mathbb{Z}_{+},\left|k_{1}-k_{2}\right| \leq 4, k \leq \min \left(k_{1}, k_{2}\right)-30, u_{k_{1}} \in$ $F_{k_{1}}$, and $v_{k_{2}} \in F_{k_{2}}$. Then

$$
\left\|P_{k}\left(\partial_{x}\left(u_{k_{1}} v_{k_{2}}\right)\right)\right\|_{N_{k}} \lesssim k_{2} 2^{k_{2}-3 k / 2} \cdot\left\|u_{k_{1}}\right\|_{F_{k_{1}}} \cdot\left\|v_{k_{2}}\right\|_{F_{k_{2}}} \cdot
$$

Proof of Lemma 7.3. Using the definitions and (2.21), the left-hand side of (7.12) is dominated by

$$
\begin{aligned}
& C \sup _{t_{k} \in \mathbb{R}} \| p(\xi, \mu)\left(\tau-\omega(\xi, \mu)+i 2^{k}\right)^{-1} \cdot 2^{k} \mathbf{1}_{I_{k}}(\xi) \cdot \sum_{|m| \leq C 2^{k_{2}-k}} \\
& \mathcal{F}\left[u_{k_{1}} \eta_{0}\left(2^{k}\left(t-t_{k}\right)\right) \gamma\left(2^{k_{2}}\left(t-t_{k}\right)-m\right)\right] * \mathcal{F}\left[v_{k_{2}} \eta_{0}\left(2^{k}\left(t-t_{k}\right)\right) \gamma\left(2^{k_{2}}\left(t-t_{k}\right)-m\right)\right] \|_{X_{k}}
\end{aligned}
$$

Using the definitions and the bounds (2.4) and (2.21), it suffices to prove that if $j_{1}, j_{2} \geq k_{2}$, and $f_{k_{i}, j_{i}}: \mathbb{R}^{3} \rightarrow \mathbb{R}_{+}$are supported in $D_{k_{i}, \infty, j_{i}}, i=1,2$, then

$$
\begin{aligned}
& 2^{k} 2^{k_{2}-k} \sum_{j \geq k} 2^{-j / 2}\left\|\mathbf{1}_{D_{k, \infty, j}} \cdot p(\xi, \mu) \cdot\left(f_{k_{1}, j_{1}} * f_{k_{2}, j_{2}}\right)\right\|_{L^{2}} \\
& \lesssim k_{2} 2^{k_{2}-3 k / 2} \cdot 2^{j_{1} / 2}\left\|p\left(\xi_{1}, \mu_{1}\right) \cdot f_{k_{1}, j_{1}}\right\|_{L^{2}} \cdot 2^{j_{2} / 2}\left\|p\left(\xi_{2}, \mu_{2}\right) \cdot f_{k_{2}, j_{2}}\right\|_{L^{2}} .
\end{aligned}
$$

Due to the rough estimate

$$
p(\xi, \mu) \lesssim 2^{2 k_{2}-2 k}\left(p\left(\xi_{1}, \mu_{1}\right)+p\left(\xi_{2}, \mu_{2}\right)\right)
$$

the bound (7.13) follows from (5.24) in the region for $j / 2 \geq 2 k_{2}-k / 2$. Therefore it remains to prove that

$$
\begin{aligned}
& 2^{-j / 2}\left\|\mathbf{1}_{D_{k, \infty, j}} \cdot p(\xi, \mu) \cdot\left(f_{k_{1}, j_{1}} * f_{k_{2}, j_{2}}\right)\right\|_{L^{2}} \\
& \lesssim 2^{-3 k / 2} \cdot 2^{j_{1} / 2}\left\|p\left(\xi_{1}, \mu_{1}\right) \cdot f_{k_{1}, j_{1}}\right\|_{L^{2}} \cdot 2^{j_{2} / 2}\left\|p\left(\xi_{2}, \mu_{2}\right) \cdot f_{k_{2}, j_{2}}\right\|_{L^{2}} .
\end{aligned}
$$

We now seek to improve (7.14). We observe that

$$
\left(\frac{\mu_{1}}{\xi_{1}}-\frac{\mu}{\xi}\right)^{2} \lesssim\left|\xi_{2}\right|^{2}+|\xi|^{-1}\left|\Omega\left(\left(\xi_{1}, \mu_{1}\right),\left(\xi_{2}, \mu_{2}\right)\right)\right|
$$


which leads to

$$
\frac{|\mu|}{|\xi|} \lesssim \frac{\left|\mu_{1}\right|}{\left|\xi_{1}\right|}+\left|\xi_{2}\right|+|\xi|^{-1 / 2}\left|\Omega\left(\left(\xi_{1}, \mu_{1}\right),\left(\xi_{2}, \mu_{2}\right)\right)\right|
$$

therefore

$$
p(\xi, \mu) \lesssim 2^{k_{2}-k} p\left(\xi_{1}, \mu_{1}\right)+2^{-3 k / 2} 2^{\max \left(j_{1}, j_{2}, j\right) / 2}
$$

Thus (7.15) follows from the bounds

$$
\left\|\mathbf{1}_{D_{k, \infty}, j} \cdot\left(f_{k_{1}, j_{1}} * f_{k_{2}, j_{2}}\right)\right\|_{L^{2}} \lesssim 2^{-k_{2}-k / 2} 2^{\left(j+j_{1}+j_{2}\right) / 2}\left\|f_{k_{1}, j_{1}}\right\|_{L^{2}}\left\|f_{k_{2}, j_{2}}\right\|_{L^{2}},
$$

and

$$
\left\|\mathbf{1}_{D_{k, \infty, j}} \cdot\left(f_{k_{1}, j_{1}} * f_{k_{2}, j_{2}}\right)\right\|_{L^{2}} \lesssim 2^{\left(j+j_{1}+j_{2}\right) / 2-\max \left(j, j_{1}, j_{2}\right) / 2}\left\|f_{k_{1}, j_{1}}\right\|_{L^{2}}\left\|f_{k_{2}, j_{2}}\right\|_{L^{2}}
$$

both of which are consequences of (5.24).

Lemma 7.4. Assume that $k_{1}, k_{2} \in \mathbb{Z}_{+},\left|k_{1}-k_{2}\right| \leq 4, k \in \mathbb{Z} \cap(-\infty, 0], k \leq$ $\min \left(k_{1}, k_{2}\right)-30, u_{k_{1}} \in F_{k_{1}}$, and $v_{k_{2}} \in F_{k_{2}}$. Then

$$
\left\|P_{k}\left(\partial_{x}\left(u_{k_{1}} v_{k_{2}}\right)\right)\right\|_{N_{k}} \lesssim\left(k_{2}-k\right) 2^{k_{2}+k / 2} \cdot\left\|u_{k_{1}}\right\|_{F_{k_{1}}} \cdot\left\|v_{k_{2}}\right\|_{F_{k_{2}}} .
$$

Proof of Lemma 7.4. As in the proof of Lemma 7.3, using the definitions and the bounds (2.4) and (2.21), it suffices to prove that if $j_{1}, j_{2} \geq k_{2}$, and $f_{k_{i}, j_{i}}: \mathbb{R}^{3} \rightarrow \mathbb{R}_{+}$ are supported in $D_{k_{i}, \infty, j_{i}}, i=1,2$, then

$$
\begin{aligned}
& 2^{k} 2^{k_{2}} \sum_{j \geq 0} 2^{-j / 2}\left\|\mathbf{1}_{D_{k, \infty}, j} \cdot p(\xi, \mu) \cdot\left(f_{k_{1}, j_{1}} * f_{k_{2}, j_{2}}\right)\right\|_{L^{2}} \\
& \lesssim\left(k_{2}-k\right) 2^{k_{2}+k / 2} \cdot 2^{j_{1} / 2}\left\|p\left(\xi_{1}, \mu_{1}\right) \cdot f_{k_{1}, j_{1}}\right\|_{L^{2}} \cdot 2^{j_{2} / 2}\left\|p\left(\xi_{2}, \mu_{2}\right) \cdot f_{k_{2}, j_{2}}\right\|_{L^{2}}
\end{aligned}
$$

Instead of (7.14) we now have

$$
p(\xi, \mu) \lesssim 2^{2 k_{2}-k}\left(p\left(\xi_{1}, \mu_{1}\right)+p\left(\xi_{2}, \mu_{2}\right)\right)
$$

which shows that the bound (7.13) follows from (5.24) for $j / 2 \geq 2 k_{2}-k / 2$. Therefore it remains to prove that

$$
\begin{aligned}
& 2^{-j / 2}\left\|\mathbf{1}_{D_{k, \infty, j}} \cdot p(\xi, \mu) \cdot\left(f_{k_{1}, j_{1}} * f_{k_{2}, j_{2}}\right)\right\|_{L^{2}} \\
& \lesssim 2^{-k / 2} \cdot 2^{j_{1} / 2}\left\|p\left(\xi_{1}, \mu_{1}\right) \cdot f_{k_{1}, j_{1}}\right\|_{L^{2}} \cdot 2^{j_{2} / 2}\left\|p\left(\xi_{2}, \mu_{2}\right) \cdot f_{k_{2}, j_{2}}\right\|_{L^{2}}
\end{aligned}
$$

We still have (7.16), but now this leads to

$$
p(\xi, \mu) \lesssim 2^{k_{2}} p\left(\xi_{1}, \mu_{1}\right)+2^{-k / 2} 2^{\max \left(j_{1}, j_{2}, j\right) / 2}
$$

Then (7.22) reduces to (7.17) and (7.18), which follow as before from (5.24).

Finally, we estimate low-frequency interactions.

Lemma 7.5. Assume $k, k_{1}, k_{2} \in(-\infty, 100] \cap \mathbb{Z}, u_{k_{1}} \in F_{k_{1}}$, and $v_{k_{2}} \in F_{k_{2}}$. Then

$$
\left\|P_{k}\left(\partial_{x}\left(u_{k_{1}} v_{k_{2}}\right)\right)\right\|_{N_{k}} \lesssim 2^{\left(k+k_{1}+k_{2}\right) / 2} \cdot\left\|u_{k_{1}}\right\|_{F_{k_{1}}} \cdot\left\|v_{k_{2}}\right\|_{F_{k_{2}}} \text {. }
$$


Proof of Lemma 7.5. As in the proof of Lemma 7.1, using the definitions and the bounds (2.4) and (2.21), it suffices to prove that if $j_{1}, j_{2} \in \mathbb{Z}_{+}$, and $f_{k_{i}, j_{i}}: \mathbb{R}^{3} \rightarrow$ $\mathbb{R}_{+}$are supported in $D_{k_{i}, \infty, j_{i}}, i=1,2$, then

$$
\begin{aligned}
& 2^{k} \sum_{j \geq 0} 2^{-j / 2}\left\|\mathbf{1}_{D_{k, \infty, j}} \cdot p(\xi, \mu) \cdot\left(f_{k_{1}, j_{1}} * f_{k_{2}, j_{2}}\right)\right\|_{L^{2}} \\
& \lesssim 2^{\left(k+k_{1}+k_{2}\right) / 2} \cdot 2^{j_{1} / 2}\left\|p\left(\xi_{1}, \mu_{1}\right) \cdot f_{k_{1}, j_{1}}\right\|_{L^{2}} \cdot 2^{j_{2} / 2}\left\|p\left(\xi_{2}, \mu_{2}\right) \cdot f_{k_{2}, j_{2}}\right\|_{L^{2}} .
\end{aligned}
$$

We may assume that $k_{1} \leq k_{2}$ (which forces $k \leq k_{2}+4$ ). We use the simple bound

$$
p(\xi, \mu) \lesssim 2^{k_{2}-k}\left(p\left(\xi_{1}, \mu_{1}\right)+p\left(\xi_{2}, \mu_{2}\right)\right),
$$

and (5.25). The bound (7.24) follows.

\section{Dyadic BILINEAR ESTimates, II}

In this section we prove several dyadic bounds which are used in the proof of Proposition 3.3 (b). We estimate first low-frequency interactions.

Lemma 8.1. Assume $k, k_{1}, k_{2} \in \mathbb{Z} \cap(-\infty, 100], u_{k_{1}} \in \bar{F}_{k_{1}}$, and $v_{k_{2}} \in F_{k_{2}}$. Then

$$
\left\|P_{k}\left(\partial_{x}\left(u_{k_{1}} v_{k_{2}}\right)\right)\right\|_{\bar{N}_{k}} \lesssim 2^{3 k / 2+k_{2} / 2} \cdot\left\|u_{k_{1}}\right\|_{\bar{F}_{k_{1}}} \cdot\left\|v_{k_{2}}\right\|_{F_{k_{2}}} \cdot
$$

Proof of Lemma 8.1. Using the definitions and the bounds (2.4) and (2.21), it suffices to prove that if $j_{1}, j_{2} \in \mathbb{Z}_{+}$, and $f_{k_{i}, j_{i}}: \mathbb{R}^{3} \rightarrow \mathbb{R}_{+}$are supported in $D_{k_{i}, \infty j_{i}}$, $i=1,2$, then

$$
\begin{aligned}
& 2^{k} \sum_{j \geq 0} 2^{-j / 2}\left\|\mathbf{1}_{D_{k, \infty, j}} \cdot\left(f_{k_{1}, j_{1}} * f_{k_{2}, j_{2}}\right)\right\|_{L^{2}} \\
& \lesssim 2^{3 k / 2+k_{2} / 2} \cdot 2^{j_{1} / 2}\left\|f_{k_{1}, j_{1}}\right\|_{L^{2}} \cdot 2^{j_{2} / 2}\left\|p\left(\xi_{2}, \mu_{2}\right) \cdot f_{k_{2}, j_{2}}\right\|_{L^{2}} .
\end{aligned}
$$

This is a direct consequence of (5.25).

We estimate now High $\times$ High $\rightarrow$ Low interactions.

Lemma 8.2. Assume $k, k_{1}, k_{2} \in \mathbb{Z}, k_{1}, k_{2} \geq \max (k-10,20), u_{k_{1}} \in \bar{F}_{k_{1}}$, and $v_{k_{2}} \in F_{k_{2}}$. Then

$$
\left\|P_{k}\left(\partial_{x}\left(u_{k_{1}} v_{k_{2}}\right)\right)\right\|_{\bar{N}_{k}} \lesssim 2^{\left(3 k_{2}-3|k|\right) / 4} \cdot\left\|u_{k_{1}}\right\|_{\bar{F}_{k_{1}}} \cdot\left\|v_{k_{2}}\right\|_{F_{k_{2}}}
$$

Proof of Lemma 8.2. As in the proof of Lemma 7.3, using the definitions and the bounds (2.4) and (2.21), it suffices to prove that if $j_{1}, j_{2} \geq k_{2}$, and $f_{k_{i}, j_{i}}: \mathbb{R}^{3} \rightarrow \mathbb{R}_{+}$ are supported in $D_{k_{i}, \infty, j_{i}}, i=1,2$, then

$$
\begin{aligned}
& 2^{k} 2^{k_{2}-k_{+}} \sum_{j \geq 0} 2^{-j / 2}\left\|\mathbf{1}_{D_{k, \infty}, j} \cdot\left(f_{k_{1}, j_{1}} * f_{k_{2}, j_{2}}\right)\right\|_{L^{2}} \\
& \lesssim 2^{\left(3 k_{2}-3|k|\right) / 4} \cdot 2^{j_{1} / 2}\left\|f_{k_{1}, j_{1}}\right\|_{L^{2}} \cdot 2^{j_{2} / 2}\left\|p\left(\xi_{2}, \mu_{2}\right) \cdot f_{k_{2}, j_{2}}\right\|_{L^{2}} .
\end{aligned}
$$

Assume first that

$$
k \geq 0
$$


Then, using (5.24), the left-hand side of (8.4) is dominated by

$$
C 2^{k_{2}} k_{2} 2^{-\left(2 k_{2}+k\right) / 2} 2^{j_{1} / 2}\left\|f_{k_{1}, j_{1}}\right\|_{L^{2}} \cdot 2^{j_{2} / 2}\left\|f_{k_{2}, j_{2}}\right\|_{L^{2}}
$$

which suffices for (8.4). Assume now that

$$
k \leq 0 \text { and } k+2 k_{2} \geq 0 .
$$

Then, using (5.24), the left-hand side of (8.4) is dominated by

$$
C 2^{k+k_{2}} k_{2} 2^{-\left(2 k_{2}+k\right) / 2} 2^{j_{1} / 2}\left\|f_{k_{1}, j_{1}}\right\|_{L^{2}} \cdot 2^{j_{2} / 2}\left\|f_{k_{2}, j_{2}}\right\|_{L^{2}},
$$

which suffices for (8.4) in view of (8.5). Finally, assume that

$$
k+2 k_{2} \leq 0
$$

Then, using (5.24), the left-hand side of (8.4) is dominated by

$$
C 2^{k+k_{2}} 2^{j_{1} / 2}\left\|f_{k_{1}, j_{1}}\right\|_{L^{2}} \cdot 2^{j_{2} / 2}\left\|f_{k_{2}, j_{2}}\right\|_{L^{2}}
$$

which suffices for (8.4) in view of (8.6).

Finally, we estimate Low $\times$ High $\rightarrow$ High interactions.

Lemma 8.3. Assume $k, k_{1}, k_{2} \in \mathbb{Z}, k \geq 20, k_{2} \leq k-10,\left|k_{1}-k\right| \leq 4, u_{k_{1}} \in \bar{F}_{k_{1}}$, and $v_{k_{2}} \in F_{k_{2}}$. Then

$$
\left\|P_{k}\left(\partial_{x}\left(u_{k_{1}} v_{k_{2}}\right)\right)\right\|_{\bar{N}_{k}} \lesssim 2^{k_{2}} \cdot\left\|u_{k_{1}}\right\|_{\bar{F}_{k_{1}}} \cdot\left\|v_{k_{2}}\right\|_{F_{k_{2}}} \text { if } k_{2} \leq 0
$$

and

$$
\left\|P_{k}\left(\partial_{x}\left(u_{k_{1}} v_{k_{2}}\right)\right)\right\|_{\bar{N}_{k}} \lesssim k_{2} 2^{-k_{2} / 2} \cdot\left\|u_{k_{1}}\right\|_{\bar{F}_{k_{1}}} \cdot\left\|v_{k_{2}}\right\|_{F_{k_{2}}} \text { if } k_{2} \geq 1 .
$$

Proof of Lemma 8.3. As in the proof of Lemma 7.1, using the definitions and the bounds (2.4) and (2.21), it suffices to prove that if $j_{1}, j_{2} \geq k$, and $f_{k_{i}, j_{i}}: \mathbb{R}^{3} \rightarrow \mathbb{R}_{+}$ are supported in $D_{k_{i}, \infty, j_{i}}, i=1,2$, then

$$
\begin{aligned}
& 2^{k} \sum_{j \geq k} 2^{-j / 2}\left\|\mathbf{1}_{D_{k, \infty}, j} \cdot\left(f_{k_{1}, j_{1}} * f_{k_{2}, j_{2}}\right)\right\|_{L^{2}} \\
& \lesssim c\left(k_{2}\right) \cdot 2^{j_{1} / 2}\left\|f_{k_{1}, j_{1}}\right\|_{L^{2}} \cdot 2^{j_{2} / 2}\left\|p\left(\xi_{2}, \mu_{2}\right) \cdot f_{k_{2}, j_{2}}\right\|_{L^{2}} .
\end{aligned}
$$

where $c\left(k_{2}\right)=2^{k_{2}}$ for $k_{2} \leq 0$, respectively $c\left(k_{2}\right)=k_{2} 2^{-k_{2} / 2}$ for $k_{2}>0$.

Consider first the case $k_{2} \leq 0$. Since $j, j_{1}, j_{1} \geq k$, the above bound is a direct consequence of (5.25).

If $k_{2} \geq 1$ then the high modulation case $j \geq k+4 k_{2}$ is obtained directly from (5.26). Therefore it remains to prove that

$$
\begin{aligned}
& \left\|\mathbf{1}_{D_{k, \infty, j}} \cdot\left(f_{k_{1}, j_{1}} * f_{k_{2}, j_{2}}\right)\right\|_{L^{2}} \\
& \lesssim 2^{-k-k_{2} / 2} \cdot 2^{\left(j+j_{1}+j_{2}\right) / 2}\left\|f_{k_{1}, j_{1}}\right\|_{L^{2}} \cdot\left\|p\left(\xi_{2}, \mu_{2}\right) \cdot f_{k_{2}, j_{2}}\right\|_{L^{2}} .
\end{aligned}
$$

This follows from (5.24). 
Lemma 8.4. Assume $k, k_{1}, k_{2} \in \mathbb{Z}, k \geq 20, k_{1} \leq k-10,\left|k-k_{2}\right| \leq 4, u_{k_{1}} \in \bar{F}_{k_{1}}$, and $v_{k_{2}} \in F_{k_{2}}$. Then

$$
\left\|P_{k}\left(\partial_{x}\left(u_{k_{1}} v_{k_{2}}\right)\right)\right\|_{\bar{N}_{k}} \lesssim 2^{-k_{1} / 2} k_{2} \cdot\left\|u_{k_{1}}\right\|_{\bar{F}_{k_{1}}} \cdot\left\|v_{k_{2}}\right\|_{F_{k_{2}}}
$$

Proof of Lemma 8.4. As in the proof of Lemma 7.1, using the definitions and the bounds (2.4) and (2.21), it suffices to prove that if $j_{1}, j_{2} \geq 0$, and $f_{k_{i}, j_{i}}: \mathbb{R}^{3} \rightarrow \mathbb{R}_{+}$ are supported in $D_{k_{i}, \infty, j_{i}}, i=1,2$, then

$$
\begin{aligned}
& 2^{k} \sum_{j \geq 0} 2^{-j / 2}\left\|\mathbf{1}_{D_{k, \infty}, j} \cdot\left(f_{k_{1}, j_{1}} * f_{k_{2}, j_{2}}\right)\right\|_{L^{2}} \\
& \lesssim 2^{-k_{1} / 2} k_{2} \cdot 2^{j_{1} / 2}\left\|f_{k_{1}, j_{1}}\right\|_{L^{2}} \cdot 2^{j_{2} / 2}\left\|p\left(\xi_{2}, \mu_{2}\right) \cdot f_{k_{2}, j_{2}}\right\|_{L^{2}} .
\end{aligned}
$$

Using (5.24), the left-hand side of (8.12) is dominated by

$$
2^{k} k_{2} \cdot 2^{-\left(2 k+k_{1}\right) / 2}\left\|f_{k_{1}, j_{1}}\right\|_{L^{2}} \cdot\left\|f_{k_{2}, j_{2}}\right\|_{L^{2}}
$$

which suffices.

\section{REFERENCES}

[1] M. Ben-Artzi and J.-C. Saut, Uniform decay estimates for a class of oscillatory integrals and applications, Differential Integral Equations 12 (1999), 137-145.

[2] J. L. Bona and R. Smith, The initial-value problem for the Korteweg-de Vries equation, Philos. Trans. Roy. Soc. London Ser. A 278 (1975), 555-601.

[3] J. Bourgain, Fourier transform restriction phenomena for certain lattice subsets and applications to nonlinear evolution equations II. The KdV-equation, Geom. Funct. Anal. 3 (1993), 209-262.

[4] J. Bourgain, On the Cauchy problem for the Kadomstev-Petviashvili Equation, Geom. Funct. Anal. 3 (1993), 315-341.

[5] M. Christ, J. Colliander, and T. Tao, A priori bounds and weak solutions for the nonlinear Schrödinger equation in Sobolev spaces of negative order, Preprint (2006).

[6] J. Colliander, A. D. Ionescu, C. E. Kenig, and G. Staffilani, Weighted low-regularity solutions of the KP-I initial-value problem, Preprint (2007).

[7] A. D. Ionescu and C. E. Kenig, Local and global well-posedness of periodic KP-I equations, Preprint (2005).

[8] R. J. Iorio and W. V. L. Nunes, On equations of KP-type, Proc. Roy. Soc. Edinburgh Sect. A 128 (1998), 725-743.

[9] C. E. Kenig, G. Ponce, and L. Vega, Well-posedness and scattering results for the generalized Korteweg-de Vries equation via the contraction principle. Comm. Pure Appl. Math. 46 (1993), 527-620.

[10] C. E. Kenig, G. Ponce, and L. Vega, The Cauchy problem for the Korteweg-de Vries equation in Sobolev spaces of negative indices. Duke Math. J. 71 (1993), 1-21.

[11] C. E. Kenig, On the local and global well-posedness theory for the KP-I equation, Ann. Inst. H. Poincaré Anal. Non Linéaire 21 (2004), 827-838.

[12] S. Klainerman and M. Machedon, Space-time estimates for null forms and the local existence theorem, Comm. Pure Appl. Math. 46 (1993), 1221-1268.

[13] H. Koch and D. Tataru, A-priori bounds for the 1-d cubic NLS in negative Sobolev spaces, Preprint (2006). 
[14] L. Molinet, J.-C. Saut, and N. Tzvetkov, Well-posedness and ill-posedness results for the Kadomtsev-Petviashvili-I equation, Duke Math. J. 115 (2002), 353-384.

[15] L. Molinet, J.-C. Saut, and N. Tzvetkov, Global well-posedness for the KP-I equation, Math. Ann. 324 (2002), 255-275.

[16] L. Molinet, J.-C. Saut, and N. Tzvetkov, Correction: Global well-posedness for the KP-I equation, Math. Ann. 328 (2004), 707-710.

[17] J.-C. Saut, Remarks on the generalized Kadomstev-Petviashvili equations, Indiana Univ. Math. J. 42 (1993), 1011-1026.

[18] H. Takaoka and N. Tzvetkov, On the local regularity of the Kadomtsev-Petviashvili-II equation, Int. Math. Res. Not. 2001 (2001), 77-114.

[19] D. Tataru, Local and global results for wave maps I, Comm. Partial Differential Equations 23 (1998), 1781-1793.

UNIVERSITY OF WISCONSIN-MADISON

E-mail address: ionescu@math.wisc.edu

University OF Chicago

E-mail address: cek@math.uchicago.edu

University of CALIFORNIA-BERKELEY

E-mail address: tataru@math. berkeley.edu 